Pacific Journal of Mathematics

THE PICARD NUMBERS OF ELLIPTIC SURFACES WITH 


\title{
THE PICARD NUMBERS OF ELLIPTIC SURFACES WITH MANY SYMMETRIES
}

\author{
Peter F. STILler
}

In this paper we compute the Picard numbers of several families of elliptic surfaces (see Example 1, $\S 5$ for a typical result.) This is equivalent to the difficult problem of determining the rank of the Mordell-Weil group of certain elliptic curves over function fields. Our method is to study the action induced by automorphisms of these surfaces on a relevant part of the cohomology. The cohomology classes are represented by certain inhomogeneous differential equations-our so-called inhomogeneous de Rham cohomology - where the effect of the action is easily understood.

1. An overview. A complex surface is said to be elliptic if it can be mapped onto a curve in such a way that the general fiber is a curve of genus one (see Kodaira [9] and [10]). In this paper we focus on computing the Picard number of certain surfaces of this type. Recall that the Picard number is defined to be the rank of the Néron-Severi group of the surface - that is, the group of divisors modulo algebraic equivalence-which is known to be a finitely generated abelian group.

Let $E$ be an elliptic surface and denote by $\pi: E \rightarrow X$ a projection of $E$ onto a curve $X$ with generic fiber $E^{\text {gen }}$ a curve of genus one over the function field $K(X)$ of $X$. We shall assume that $\pi: E \rightarrow X$ has a section $\circ: X \rightarrow E, \pi \circ \circ=1_{X}$, that the $J$-invariants of the fibers are not constant, and that there are no exceptional curves of the first kind in the fibers. Let $S \subset X$ be the finite set of points at which the family $E / X$ degeneratesthat is, where $\pi^{-1}(s)$ fails to be an elliptic curve. (Note that there are no multiple fibers.) The degenerate fiber types are classified (see Kodaira [9]) and we shall label the types following Kodaira. We denote by $\operatorname{NS}(E)$ the Néron-Severi group of $E$ and by $\rho_{E}$ its rank which is called the Picard number of $E$.

The group $\mathrm{NS}(E)$ is naturally a subgroup of $H^{2}(E, \mathbf{Z})$ - both are torsion free in our case (see Cox and Zucker [1]) - and includes in $H^{1}\left(E, \Omega_{E}^{1}\right)$ the $(1,1)$ part of the Hodge decomposition of the cohomology, 
$H^{2}(E, \mathrm{C})$, of $E$ :

$$
\begin{aligned}
& H^{2}(E, \mathbf{Z}) \\
& C \quad C \\
& \mathrm{NS}(E) \\
& H^{2}(E, \mathbf{C}) \\
& \text { ค } H^{1}\left(E, \Omega_{E}^{1}\right) \text { C }
\end{aligned}
$$

Of course, by the Lefschetz theorem on $(1,1)$-classes (Griffiths and Harris [5]), NS $(E)=H^{2}(E, \mathbf{Z}) \cap H^{1}\left(E, \Omega_{E}^{1}\right)$ in $H^{2}(E, \mathbf{C})$. So $N S(E)$ is just the group of topological complex line bundles which admit an analytic structure.

In order to compute the Picard number, as we do in the examples below, it will be necessary to have a thorough understanding of the structure of the cohomology of our surfaces and an effective way of representing it.

We begin by considering the Leray spectral sequence for $\pi: E \rightarrow X$ and the constant sheaf $\mathbf{Q}$ on $E$ :

$$
E_{2}^{p q}=H^{p}\left(X, R^{q} \pi_{*} \mathbf{Q}\right) \Rightarrow H^{*}(E, \mathbf{Q}) .
$$

This is well-known to degenerate at $E_{2}$. Thus we have a filtration of the rational cohomology

$$
0 \subset F_{\mathbf{Q}}^{2} \subset F_{\mathbf{Q}}^{1} \subset F_{\mathbf{Q}}^{0}=H^{2}(E, \mathbf{Q})
$$

with

$$
F_{\mathbf{Q}}^{1}=\operatorname{ker}\left(H^{2}(E, \mathbf{Q}) \rightarrow H^{0}\left(X, R^{2} \pi_{*} \mathbf{Q}\right)\right)
$$

consisting of classes which restrict to zero on the fibers; with

$$
F_{\mathbf{Q}}^{2}=\operatorname{im}\left(H^{2}(X, \mathbf{Q}) \stackrel{\pi^{*}}{\rightarrow} H^{2}(E, \mathbf{Q})\right)=\mathbf{Q}\left[E_{x_{0}}\right], \quad E_{x_{0}}=\pi^{-1}\left(\overline{x_{0}}\right),
$$

generated by the cohomology class of the fiber, and $F_{\mathbf{Q}}^{1} / F_{\mathbf{Q}}^{2} \cong$ $H^{1}\left(X, R^{1} \pi_{*} \mathbf{Q}\right)$. Moreover, the Hodge decomposition of the complex cohomology $H^{*}(E, \mathrm{C})$ induces a Hodge structure on the filtration quotients coming from the Leray spectral sequence over $\mathbf{C}$ (Cox and Zucker [1]). The most interesting term is $H^{1}\left(X, R^{1} \pi_{*} \mathrm{C}\right)$ and we express the Hodge structure on it by writing

$$
H^{1}\left(X, R^{1} \pi_{*} \mathrm{C}\right)=H^{2,0} \oplus H^{1,1} \oplus H^{0,2} .
$$

Notice that $H^{2,0}$ is in fact all of $H^{0}\left(E, \Omega_{E}^{2}\right)$. 
Now that we understand the structure of the cohomology of $E$ over $X$, we will need to know where the algebraic cycles fit in. We first describe $\mathrm{NS}(E)$.

Proposition 1.2 (Shioda [14]). Let $E_{x_{0}}=\pi^{-1}\left(x_{0}\right)$ denote a good fiber over some point $x_{0} \in X-S$; let $D_{s, 0}, \ldots, D_{s, l}, \ldots, D_{s, m_{s}-1}, s \in S$, denote the (reduced) irreducible components ( $m_{s}$ in number) of the singular fiber $\pi^{-1}(s)$ with $D_{s, 0}$ being the unique component meeting the image of the zero section $o$; and let $s_{1}, \ldots, s_{r}$ be sections which modulo torsion form a basis for the group of sections with $t_{1}, t_{2}$ sections of order $e_{1}, e_{2}$ respectively, $1 \leq e_{\beta}$, $e_{2} \mid e_{1}$, generating the torsion subgroup of order $e_{1} e_{2}$. The Néron-Severi group NS( $E)$ of the elliptic surface $E$ is generated by the divisiors:

$$
\begin{gathered}
E_{x_{0}}, \quad D_{s, i} \quad\left(1 \leq i \leq m_{s}-1, s \in S\right), \quad(0) \\
D_{\alpha}=\left(s_{\alpha}\right)-(0) \quad(1 \leq \alpha \leq r) \quad \text { and } \quad D_{\beta}^{\prime}=\left(t_{\beta}\right)-(0) \quad(\beta=1,2)
\end{gathered}
$$

where ( ) indicates the curve on $E$ which is the image of the section. The fundamental relations between these generators are (at most) the two relations:

$$
e_{\beta} D_{\beta}^{\prime} \sim e_{\beta}\left(D_{\beta}^{\prime} \cdot(o)\right) E_{x_{0}}+\sum_{s \in S}\left(D_{s, 1}, \ldots, D_{s, m_{s}-1}\right) e_{\beta} A_{s}^{-1}\left(\begin{array}{c}
\left(D_{\beta}^{\prime} \cdot D_{s, 1}\right) \\
\vdots \\
\left(D_{\beta}^{\prime} \cdot D_{s, m_{s}-1}\right)
\end{array}\right)
$$

for $\beta=1,2$ where $~$ indicates algebraic equivalence and where $A_{s}$ is the $\left(m_{s}-1\right) \times\left(m_{s}-1\right)$ negative definite matrix whose $(i, j)$-entry is $\left(D_{s, l}\right.$. $\left.D_{s, J}\right)$ the intersection number of the two divisors on $E$.

Proof. See Shioda [14].

COROLlary 1.3. The Picard number $\rho_{E}$ of the surface $E$ is given by

$$
\rho_{E}=r_{E}+2+\sum_{s \in S}\left(m_{s}-1\right),
$$

where $r_{E}$ is the rank of the group of sections of $E$ over $X$.

In the examples below we shall focus on computing $r_{E}$, but as this formula shows, we can then determine $\rho_{E}$ provided we know the types of the degenerate fibers. The latter are generally easy to determine in actual practice. On the other hand, computing $r_{E}$ is usually very difficult. 
We consider the rational span of the algebraic cycles that lie in the $i$ th level of the Leray filtration

$$
V_{\mathbf{Q}}^{i} \stackrel{\text { defn }}{=}\left(\mathrm{NS}(E) \otimes_{\mathbf{z}} \mathbf{Q}\right) \cap F_{\mathbf{Q}}^{i}
$$

and the resulting subspace of $H^{1}\left(X, R^{1} \pi_{*} \mathbf{Q}\right)$

$$
W_{\mathbf{Q}}=V_{\mathbf{Q}}^{1} / V_{\mathbf{Q}}^{2}=\frac{\left(\mathrm{NS}(E) \otimes_{\mathbf{Z}} \mathbf{Q}\right) \cap F_{\mathbf{Q}}^{1}}{F_{\mathbf{Q}}^{2}} \subset H^{1}\left(X, R^{1} \pi_{*} \mathbf{Q}\right),
$$

which makes sense because $F_{\mathbf{Q}}^{2}=\mathbf{Q}\left[E_{x_{0}}\right]$.

Proposition 1.4. $\operatorname{dim}_{\mathbf{Q}} W_{\mathbf{Q}}=r_{E}$.

Proof. This is a straightforward consequence of Proposition 1.2 above and a result of Cox and Zucker, Proposition 1.5, below.

Proposition 1.5 (Cox and Zucker [1]). Let $s$ be any section of $E$ over $X$, then there exists a rational linear combination $\Sigma_{s \in S} D_{s}$ of the components of the bad fibers $\left(D_{s}=\sum_{i=0}^{m_{s}-1} a_{s, i} D_{s, i}, a_{s, i} \in \mathbf{Q}\right)$ so that $[(s)-(0)+$ $\sum_{s \in S} D_{s}$ ] lies in $F_{\mathbf{Q}}^{1}$. Moreover each $D_{s}$ is unique up to a rational multiple of the total fiber $E_{s}=\pi^{-1}(s)$ so that $\left[(s)-(\mathcal{O})+\sum_{s \in S} D_{s}\right]$ gives a well-defined class in $H^{1}\left(X, R^{1} \pi_{*} \mathbf{Q}\right)$.

Proof. See Cox and Zucker [1].

2. An inhomogeneous de Rham cohomology. In order to actually make computations our surface $E$ must be given to us in a manageable form-the most convenient being a Weierstrass model for the generic fiber $E^{\text {gen }}$ as an elliptic curve over the function field $K(X)$ of the base curve $X$ :

$$
Y^{2}=4 X^{3}-g_{2} X-g_{3}, \quad g_{2}, g_{3} \in K(X) .
$$

$E$ is then just the relatively minimal compactification of the Néron model of $E^{\text {gen }} / K(X)$ (see Néron [11]). (Note that the group of sections corresponds to the Mordell-Weil group of $K(X)$-rational points on $E^{\text {gen }}$.)

Choose a non-trivial derivation $d / d x$ on $K(X)$ by selecting a nonconstant function $x \in K(X)$. This function will provide us with a local parameter except at a finite set of points. Denote by $\Lambda$ the linear second order differential operator with regular singular points

$$
\Lambda=\frac{d^{2}}{d x^{2}}+P \frac{d}{d x}+Q, \quad P, Q \in K(X),
$$


which annihilates the periods of $d X / Y$ for the model (2.1) above. This is the so-called Picard-Fuchs equation (Gauss-Manin connection) and in our case its monodromy can be taken to lie in $\mathrm{SL}_{2}(\mathbf{Z})$ - see Deligne [2], Katz and Oda [8] and/or Griffiths [4] for the Gauss-Manin connection in general and Stiller [15] for details in this specific case. One can say a great deal about $\Lambda$-in fact it is very easy to compute given the model (2.1) - and we shall briefly recall a few facts.

First, if we write (see 2.1)

$$
g_{2}=\lambda^{-4} \frac{27 J}{J-1}, \quad g_{3}=\lambda^{-6} \frac{27 J}{J-1},
$$

where $J=\left(g_{2}^{3} /\left(g_{2}^{3}-27 g_{3}^{2}\right)\right)$ and $\lambda=\sqrt{g_{2} / g_{3}}$ is an algebraic function on $X$ with $\lambda^{2} \in K(X)$, then

$$
\Lambda=\frac{d^{2}}{d x^{2}}+P \frac{d}{d x}+Q
$$

where

$$
\begin{aligned}
P= & \frac{(d j / d x)^{2}-J d^{2} J / d x^{2}}{J d J / d x}-\frac{d}{d x} \log \lambda^{2} \\
Q= & \frac{(d J / d x)^{2}(31 / 144 J-1 / 36)}{J^{2}(J-1)^{2}}-\frac{(d J / d x)^{2}-J d^{2} J / d x^{2}}{J d J / d x} \\
& \cdot \frac{d}{d x} \log \lambda+\frac{\left(d^{2} \lambda / d x^{2}\right)}{\lambda}+2\left(\frac{(d \lambda / d x)}{\lambda}\right)^{2}
\end{aligned}
$$

Second, the equation $\Lambda$ depends on both the choice of model and the choice of "parameter". If, for example, we were to choose another model

$$
Y^{2}=4 X^{3}-\tilde{g}_{2} X-\tilde{g}_{3}, \quad \tilde{g}_{2}, \tilde{g}_{3} \in K(X),
$$

then for some $g \in K(X)^{*}$ we would have

$$
\tilde{g}_{2}=g^{-4} g_{2}, \quad \tilde{g}_{3}=g^{-6} g_{3} .
$$

In effect, the periods have been multiplied by $g$ and we find that the new equation is the "twist" $\Lambda_{g}$ of the original-that is, the new equation has for its solutions $g$ times those of the original equation $\Lambda$. Once the model is fixed however, the equation $\Lambda$ is uniquely determined (apart from changing parameter) and we can select a basis $\omega_{1}, \omega_{2}$ for its space of solutions which precisely gives the periods of $d X / Y$ and such that the imaginary part of the quotient is positive, $\operatorname{Im}\left(\omega_{1} / \omega_{2}\right)>0$, on some Zariski open subset of $X$. Note that $\Lambda$ may have singularities outside the set $S$ which is the support of the singular fibers. 
Third, the differential equation $\Lambda$ determines the surface $E$ up to generic isogeny (see Stiller [15] and [16]). Fortunately, all the important numerical invariants $\left(\rho_{E}, r_{E}, q, p_{g}\right.$, the betti numbers) are preserved under generic isogeny, even though the fiber types may change (see Stiller [16]). As this includes the Picard number, we might reasonably expect that $\rho_{E}$ can be determined from $\Lambda$. This is the motivation behind the inhomogeneous de Rham cohomology that we introduce below.

Finally we remark that the local monodromy of $\Lambda$ is related to the type of fiber present at a particular point-see Kodaira [9].

We begin with some definitions. Given the elliptic surface $E$ over $X$, we choose a model for its generic fiber as in (2.1) above and denote by $\Lambda$ the operator annihilating the periods of $d X / Y$ for this model.

Definition 2.2. We say that an inhomogeneous equation

$$
\Lambda f=Z, \quad Z \in K(X),
$$

is exact if it has a global single-valued solution. Such a solution is necessarily a rational function (because it is annihilated by the composite operator $((d / d x)-(d / d x) \log Z) \circ \Lambda$ which has regular singularities) so that $Z=\Lambda Z^{\prime}$ for some $Z^{\prime} \in K(X)$. We will denote the space of exact equations by $\Lambda K(X)$.

DefinItion 2.3. We say that an inhomogeneous equation

$$
\Lambda f=Z, \quad Z \in K(X),
$$

is locally exact if for every point $p \in X$ the equation restricted to a small open neighborhood $U_{p}$ of $p$ has a single-valued solution. Such a solution is necessarily meromorphic in $U_{p}$. We will denote the space of locally exact equations by $L_{\Lambda}^{\text {para }}$ (for reasons that will become clear later).

Clearly exact implies locally exact so that we can form the quotient space. (In both cases, we view $\Lambda K(X)$ and $L_{\Lambda}^{\text {para }}$ as subspaces of $K(X)$ via the right-hand side, $Z$, of the equation.)

DEFINITION 2.4. We define the inhomogeneous de Rham cohomology, $H_{\mathrm{DR}}^{1}$, to be the complex vector space

$$
H_{\mathrm{DR}}^{1} \stackrel{\text { defn }}{=} L_{\Lambda}^{\text {para }} / \Lambda K(X)=\frac{\text { locally exact } .}{\text { exact }}
$$


We remark that this definition is essentially independent of the choices made above. If we choose another model as in (2.1a), then for an inhomogeneous equation $\Lambda f=Z, Z \in K(X)$, we have the relation

$$
\Lambda_{g}(g f)=g Z \text {, }
$$

where $g \in K(X)$ is the factor of homothety in the change of model. We can then identify $L_{\Lambda}^{\text {para }} \cong L_{\Lambda_{g}}^{\text {para }}$, etc., by simple (linear) equivalence sending $Z$ to $g Z$. In addition if we change parameter from $x$ to say $t$, then we have the relation

$$
\Lambda^{\mathrm{new}} f=Z\left(\frac{d x}{d t}\right)^{2}
$$

where $\Lambda^{\text {new }}$ is the operator expressed in terms of $t$ :

$$
\Lambda^{\text {new }}=\frac{d^{2}}{d t^{2}}+P^{\text {new }} \frac{d}{d t}+Q^{\text {new }}
$$

and $f$ is the original solution $\Lambda f=Z$. Thus the quadratic differential $Z(d x)^{2}$ is independent of parameter-a remark that will be useful in our calculations.

In order to understand the conditions imposed by local exactness we reformulate it in terms of residues.

Proposition 2.5. An equation $\Lambda f=Z$ is locally exact if and only if for every $p \in X$ and every single-valued (necessarily meromorphic) solution $\tilde{\omega}$ of $\Lambda$ at $p$ the differential

$$
\frac{\tilde{\omega} Z}{W} d x, \quad \text { W the Wronskian, }
$$

has zero residue. (Thus locally exact might also be properly called second kind.)

Proof. In a small enough neighborhood $U_{p}$ of $p$, we can select a base point $x_{0}$, other than $p$ itself, and via variation of parameters, write our multivalued solution $f$ in the form

$$
\begin{aligned}
& f=\left(\int_{x_{0}}^{x}-\frac{\omega_{2} Z}{w} d x+c_{1}\right) \omega_{1}+\left(\int_{x_{0}}^{x} \frac{\omega_{1} Z}{W} d x+c_{2}\right) \omega_{2}, \\
& c_{1}, c_{2} \in \mathbf{C}, x \in U_{p}-\{p\},
\end{aligned}
$$


where $\omega_{1}, \omega_{2}$ are a basis for the space of solutions to $\Lambda$ at $x_{0}$ (usually normalized to give the periods of $d X / Y$ and so that $\left.\operatorname{Im} \omega_{1} / \omega_{2}>0\right)$ and $W=\omega_{1}\left(d \omega_{2} / d x\right)-\omega_{2}\left(d \omega_{1} / d x\right)$ is the Wronskian. Note that we have somewhat abused notation by using $x$ for both a variable point in the upper limit of integration and as parameter.

Now consider a simple path $\gamma_{p}$ around $p$

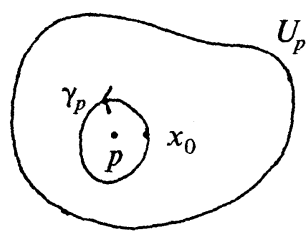

and let $M_{\gamma_{p}} \in \mathrm{SL}_{2}(\mathrm{Z})$ be the local monodromy of $\omega_{1}, \omega_{2}$ around $\gamma_{p}$. The condition on the residues is vacuous unless the local monodromy is $\left(\begin{array}{ll}1 & 0 \\ 0 & 1\end{array}\right)$, i.e. trivial, in which case all solutions of $\Gamma$ are single-valued, or unless the local monodromy is conjugate to $\left(\begin{array}{ll}1 & b \\ 0 & 1\end{array}\right)$ in $\operatorname{SL}_{2}(Z)$ in which case there will be a one-dimensional space of single-valued solutions. (In terms of the surface $E$, the fiber will be good in the first case and have type $I_{b}, b>0$, in the second-see Kodaira [9].) We caution that $\Lambda$ may have cosingular points-i.e. points with no local monodromy but at which $\Lambda$ is singular and has (possibly) meromorphic, but single-valued, solutions.

Now assume the residue condition, then analytic continuation of $f$ around $\gamma_{p}$ gives

$$
f \rightarrow f+m_{\gamma_{p}} \omega_{1}+n_{\gamma_{p}} \omega_{2}
$$

with the periods $m_{\gamma_{p}}, n_{\gamma_{p}} \in \mathbf{C}$. One easily calculates that

$$
\left[m_{\gamma_{p}}, n_{\gamma_{p}}\right]=\left[x_{1}\left(\gamma_{p}\right), x_{2}\left(\gamma_{p}\right)\right] M_{\gamma_{p}}+\left[c_{1}, c_{2}\right]\left(M_{\gamma_{p}}-I\right)
$$

where

$$
x_{1}\left(\gamma_{p}\right)=\int_{\gamma_{p}} \frac{-\omega_{2} Z}{W} d x
$$

and

$$
x_{2}\left(\gamma_{p}\right)=\int_{\gamma_{p}} \frac{\omega_{1} Z}{W} d x
$$

Now if $M_{\gamma_{p}}-I$ is invertible, we can always choose $\left[c_{1}, c_{2}\right]$ so that $\left[m_{\gamma_{p}}, n_{\gamma_{p}}\right]=[0,0]$ and then that particular solution $f$ will be single-valued. If on the other hand $M_{\gamma_{p}}-I$ is not invertible, we will have either 
$M_{\gamma_{p}}=\left(\begin{array}{ll}1 & 0 \\ 0 & 1\end{array}\right)$ or conjugate to $\left(\begin{array}{ll}1 & b \\ 0 & 1\end{array}\right), b>0$. In the first case, $\omega_{1}$ and $\omega_{2}$ will be single-valued at $p$ and the residue conditions then imply that $\left[m_{\gamma_{p}}, n_{\gamma_{p}}\right]$ $=[0,0]$ so that every solution to $\Lambda f=Z$ at $p$ is single-valued (but perhaps meromorphic). Finally in the second case, we can assume without loss of generality that $\omega_{1}, \omega_{2}$ were chosen so that $M_{\gamma_{p}}=\left(\begin{array}{ll}1 & b \\ 0 & 1\end{array}\right), b>0$. We then have

$$
\left[m_{\gamma_{p}}, n_{\gamma_{p}}\right]=\left[x_{1}\left(\gamma_{p}\right), x_{1}\left(\gamma_{p}\right)+x_{2}\left(\gamma_{p}\right)+c_{1}\right]
$$

As $\omega_{2}$ is single-valued by assumption, the residue condition gives $x_{1}\left(\gamma_{p}\right)$ $=0$ and so by setting $c_{1}=-x_{2}\left(\gamma_{p}\right)$ we get a single-valued solution.

Conversely, if we assume that locally single-valued solutions exist, then we can check the residue conditions at the points where the local monodromy $M_{\gamma_{p}}$ is $\left(\begin{array}{ll}1 & 0 \\ 0 & 1\end{array}\right)$ or conjugate to $\left(\begin{array}{ll}1 & b \\ 0 & 1\end{array}\right), b>0$, by reversing the above argument.

We would like to identify $H_{\mathrm{DR}}^{1}$ with the relevant part of $H^{2}(E, \mathrm{C})$; namely the filtration quotient $H^{1}\left(X, R^{1} \pi_{*} \mathrm{C}\right)$. In order to do this, we offer another interpretation of $H_{\mathrm{DR}}^{1}$.

As usual let $S \subset X$ denote the set of true singularities of $\Lambda$; that is, the set of singular points where the local monodromy is non-trivial. We can define a local system $V_{\Lambda}$ (see Deligne [2]) on the Zariski open set $X_{0}=X-S$ by considering the locally constant sheaf on $X_{0}$ whose sections (in small open sets) are the single-valued solutions to $\Lambda$ (which, as we have pointed out several times, may be meromorphic). Consider now a locally exact equation $\Lambda f=Z$, and the composite third order equation

$$
\tilde{\Lambda}=\left(\frac{d}{d x}-\frac{d}{d x} \log Z\right) \circ \Lambda \text {. }
$$

The residue conditions for local exactness assure us that at every point $p \in X_{0}$ the equation $\tilde{\Lambda}$ has trivial local monodromy, i.e. that all the solutions at $p$ are single-valued. Thus we can associate to $\tilde{\Lambda}$ a local system $V_{\tilde{\Lambda}}$ as we did above for $\Lambda$ and it is clear that we have a canonical inclusion $i$ :

$$
0 \rightarrow V_{\Lambda} \stackrel{i}{\rightarrow} V_{\tilde{\Lambda}} \stackrel{\Lambda}{\rightarrow} \underline{\mathbf{C}} \rightarrow 0
$$

with cokernel the trivial local system $\underline{\mathbf{C}}$ (essentialy $\underline{\mathbf{C}} Z$ ). Thus each element of $L_{\Lambda}^{\text {para }}$ defines an extension in $\operatorname{Ext}^{1}\left(\underline{\mathbf{C}}, V_{\Lambda}\right)$ over $X_{0}$.

Definition 2.6. An extension of local systems on $X_{0} \subset X$

$$
0 \rightarrow V_{\Lambda} \rightarrow V_{\tilde{\Lambda}} \rightarrow \underline{\mathrm{C}} \rightarrow 0
$$


(or more generally) is said to be locally split if for every point $p \in X$ the extension becomes trivial (i.e. splits) on some punctured neighborhood $U_{p}-\{p\}$ of $p$.

We can now characterize $H_{\mathrm{DR}}^{1}$ as the set of locally split extensions; which we denote by 1. s. $\operatorname{Ext}^{1}\left(\underline{\mathbf{C}}, V_{\Lambda}\right)$.

Proposition 2.7. The inhomogeneous de Rham cohomology $H_{D R}^{1}$ is naturally isomorphic to the space of locally split extensions of $\underline{\mathbf{C}}$ by $V_{\Lambda}$, 1.s.Ext ${ }^{1}\left(\underline{\mathbf{C}}, V_{\Lambda}\right)$.

Proof. As above each locally exact inhomogeneous equation $\Lambda f=Z$ gives rise to an extension so that we have a map

$$
\begin{aligned}
L_{\Lambda}^{\text {para }} & \rightarrow \operatorname{Ext}^{1}\left(\underline{\mathbf{C}}, V_{\Lambda}\right), \\
\Lambda f=Z & \mapsto 0 \rightarrow V_{\Lambda} \stackrel{i}{\rightarrow} V_{\tilde{\Lambda}} \stackrel{\Lambda}{\rightarrow} \underline{\mathbf{C}} \rightarrow 0 .
\end{aligned}
$$

The extension can be characterized by the monodromy of $\tilde{\Lambda}$ with respect to the specific basis $f, \omega_{1}, \omega_{2}$ for any choice of particular solution $f$. That monodromy takes the form

$$
\gamma \rightarrow\left(\begin{array}{lll}
1 & m_{\gamma} & n_{\gamma} \\
0 & & \\
& M_{\gamma} & \\
0 & & \gamma \in \pi_{1}\left(X_{0}, x_{0}\right),
\end{array}\right.
$$

where $M_{\gamma} \in \mathrm{SL}_{2}(\mathbf{Z})$ is the monodromy of $\Lambda$ with respect to $\omega_{1}, \omega_{2}$ around $\gamma$ and $f \rightarrow f+m_{\gamma} \omega_{1}+n_{\gamma} \omega_{2}$ around $\gamma$ so that $m_{\gamma}, n_{\gamma}$ are the periods as before. Given a point $p \in X$ we can always choose a branch of $f$ which is locally single-valued at $p$ because we have assumed local exactness. Thus we can arrange that in a punctured neighborhood of $p$ the monodromy will be

$$
\gamma_{p} \rightarrow\left(\begin{array}{ccc}
1 & 0 & 0 \\
0 & & \\
& M_{\gamma_{p}} & \\
0 & &
\end{array}\right)
$$

for a simple circuit around $p$. This means that our extension is locally split. Thus we have that our map goes into locally split extensions

$$
L_{\Lambda}^{\text {para }} \rightarrow 1 . s . \operatorname{Ext}^{1}\left(\underline{\mathbf{C}}, V_{\Lambda}\right) \subset \operatorname{Ext}^{1}\left(\underline{\mathbf{C}}, V_{\Lambda}\right) .
$$


It is obvious that an exact equation gives rise to the trivial (globally split) extension on $X_{0}$, and conversely. Thus we have an injection

$$
H_{\mathrm{DR}}^{1} \hookrightarrow \text { 1.s. } \operatorname{Ext}^{1}\left(\underline{\mathbf{C}}, V_{\Lambda}\right) \subset \operatorname{Ext}^{1}\left(\underline{\mathbf{C}}, V_{\Lambda}\right) .
$$

Finally any extension over $X_{0}$

$$
0 \rightarrow V_{\Lambda} \rightarrow V \rightarrow \underline{\mathbf{C}} \rightarrow 0
$$

can be given by some differential equation $\tilde{\Lambda}, V=V_{\tilde{\Lambda}}$, (see Deligne [2]) and it is clear that by a proper choice of frame we can arrange that the solutions of $\Lambda$ also be solutions of $\tilde{\Lambda}$. We then choose any section of $V$ lifting $1 \in \underline{\mathbf{C}}$, view it as a solution of $\tilde{\Lambda}$ via our choice of frame and apply $\Lambda$ to it to get a $Z$. Moreover, if the extension was locally split it is easy to see that

$$
\Lambda f=Z
$$

is then locally exact. In other words, every locally split extension over $X_{0}$ arises from some inhomogeneous equation which is locally exact. Thus our map is surjective and

$$
H_{\mathrm{DR}}^{1} \cong 1 . \mathrm{s} . \mathrm{Ext}^{1}\left(\underline{\mathbf{C}}, V_{\Lambda}\right)
$$

We remark that geometrically the third order equation $\tilde{\Lambda}$ will be the differential equation satisfied by a normal function in suitable cases.

To complete our identification of $H_{\mathrm{DR}}^{1}$ with $H^{1}\left(X, R^{1} \pi_{*} \mathrm{C}\right)$, note that on $X_{0}$ the group $\operatorname{Ext}^{1}\left(\underline{\mathbf{C}}, V_{\Lambda}\right)$ is isomorphic to $H^{1}\left(\pi_{1}\left(X_{0}, x_{0}\right), V_{\Lambda, x_{0}}\right)$ where $H^{1}$ is the ordinary group cohomology and $\pi_{1}$ acts on the fiber of $V_{\Lambda}$ at $x_{0}$ via the monodromy. (In fact $H^{1}$ is often defined to be $\operatorname{Ext}_{A}^{1}\left(A, V_{x_{0}}\right)$ where $A$ is the group algebra/C of $\pi_{1}$ and $V_{x_{0}}$ is viewed as an $A$-module in the obvious way.) In terms of cocycles: given an extension on $X_{0}$

$$
0 \rightarrow V_{\Lambda} \rightarrow V \rightarrow \underline{\mathbf{C}} \rightarrow 0
$$

or equivalently an inhomogeneous equation

$$
\Lambda f=Z \text {, }
$$

the assignment

$$
\gamma \in \pi_{1}\left(X_{0}, x_{0}\right) \rightarrow\left[m_{\gamma}, n_{\gamma}\right] \in \mathbf{C}^{2},
$$

where $m_{\gamma}$ and $n_{\gamma}$ are the periods of $f$, gives rise to a cocycle in group cohomology - it satisfies the usual relation

$$
\left[m_{\gamma \tau}, n_{\gamma \tau}\right]=\left[m_{\gamma}, n_{\gamma}\right] M_{\tau}+\left[m_{\tau}, n_{\tau}\right]
$$


It is easily checked that such an assignment is a coboundary, i.e.

$$
\left[m_{\gamma}, n_{\gamma}\right]=\left[c_{1}, c_{2}\right]\left(M_{\gamma}-I\right) \text { for every } \gamma \in \pi_{1}\left(X_{0}, x_{0}\right) \text {, }
$$

if and only if $\Lambda f=Z$ is exact.

Now on $X_{0}$ the sheaf (local system) $\left.R^{1} \pi_{*} \mathrm{C}\right|_{X_{0}}=R^{1}\left(\left.\pi\right|_{\pi^{-1}\left(X_{0}\right)}\right)_{*} \mathrm{C}$ is just $V_{\Lambda}$, and it follows that

$$
H^{1}\left(X_{0},\left.R^{1} \pi_{*} \mathbf{C}\right|_{X_{0}}\right) \cong H^{1}\left(\pi_{1}\left(X_{0}, x_{0}\right), V_{\Lambda, x_{0}}\right) .
$$

The left-hand side is the cohomology with "twisted coefficients", and the isomorphism results from the fact that $X_{0}$ is a $K(\pi, 1)$. Thus

$$
\operatorname{Ext}^{1}\left(\underline{\mathrm{C}}, V_{\Lambda}\right) \cong H^{1}\left(X_{0},\left.R^{1} \pi_{*}\right|_{X_{0}}\right)
$$

Considering the inclusion $i: X_{0} \rightarrow X$, we see that the exact sequence of low order terms in the Leray spectral sequence for $\left.R^{1} \pi_{*} \mathbf{C}\right|_{X_{0}}$ is $0 \rightarrow H^{1}\left(X, i_{*}\left(\left.R^{1} \pi \mathbf{C}\right|_{X_{0}}\right)\right) \rightarrow H^{1}\left(X_{0},\left.R^{1} \pi_{*} \mathbf{C}\right|_{X_{0}}\right) \rightarrow H^{0}\left(X, R^{1} i_{*}\left(\left.R^{1} \pi_{*} \mathbf{C}\right|_{X_{0}}\right)\right)$,

but it is well known that $i_{*}\left(\left.R^{1} \pi_{*} \mathrm{C}\right|_{X_{0}}\right)=R^{1} \pi_{*} \mathrm{C}$ (see Kodaira [9] or Cox and Zucker [1]) and moreover that the last sheaf $R^{1} i_{*}\left(\left.R^{1} \pi_{*} \mathrm{C}\right|_{X_{0}}\right)=R^{1} i_{*} V_{\Lambda}$ is supported at only a finite number of points in $S$. Thus the sequence reads

$$
0 \rightarrow H^{1}\left(X, R^{1} \pi_{*} \mathbf{C}\right) \rightarrow H^{1}\left(X_{0}, R^{1} \pi_{*} \mathbf{C}_{X_{0}}\right) \stackrel{\alpha}{\rightarrow} H^{0}\left(X, R^{1} i_{*} V_{\Lambda}\right)
$$

and we see that $H^{1}\left(X, R^{1} \pi_{*} \mathrm{C}\right)$ is a subgroup of $H^{1}\left(X_{0},\left.R^{1} \pi_{*} \mathrm{C}\right|_{X_{0}}\right) \cong$ $\operatorname{Ext}^{1}\left(\underline{\mathbf{C}}, V_{\Lambda}\right)$ determined by conditions at some of the points in $S$. As expected we have:

THEOREM 2.8. The inhomogeneous de Rham cohomology $H_{\mathrm{DR}}^{1}$ is naturally isomorphic to $H^{1}\left(X, R^{1} \pi_{*} \mathrm{C}\right)$.

Proof. We have see that $H^{1}\left(X_{0},\left.R^{1} \pi_{*} \mathrm{C}\right|_{X_{0}}\right)$ can be identified with the ordinary group cohomology $H^{1}\left(\pi_{1}\left(X_{0}, x_{0}\right), V_{\Lambda, x_{0}}\right)$ and it is known that $H^{1}\left(X, R^{1} \pi_{*} \mathrm{C}\right)$ corresponds to the parabolic cohomology $H_{\text {para }}^{1}\left(\pi_{1}\left(X_{0}, x_{0}\right), V_{\Lambda, x_{0}}\right)$ (think of $\pi_{1}$ as a Fuchsian group $\Gamma \subset \operatorname{PSL}_{2}(\mathbf{R})$ acting on $\mathfrak{h}$ the complex upper-half plane which is the universal cover of $X_{0}$ ) - see Zucker [19]. In fact one has the following lemma due to Zucker: 
Let $V$ be any locally constant sheaf on the non-singular algebraic curve $X_{0}$, with fundamental group $\Gamma \subset \operatorname{PSL}_{2}(\mathbf{R}), \tilde{V}$ the associated $\Gamma$ module, and

$$
i: X_{0} \rightarrow X
$$

the inclusion of $X_{0}$ in its smooth completion. Then there is a commutative diagram with exact rows:

$$
\begin{array}{ccccccc}
0 & \rightarrow & H^{1}\left(X, i_{*} V\right) & \rightarrow & H^{1}\left(X_{0}, V\right) & \rightarrow & \bigoplus_{s \in S} H^{1}\left(\Delta^{*}(s), V\right) \\
& & & & & & \\
0 & \rightarrow & H_{\mathrm{para}}^{1}(\Gamma, \tilde{V}) & \rightarrow & H^{1}(\Gamma, \tilde{V}) & \rightarrow & \bigoplus_{\Gamma_{0}} H^{1}\left(\Gamma_{0}, \tilde{V}\right)
\end{array}
$$

where $\Gamma_{0}$ runs over the conjugacy classes of parabolic subgroups of $\Gamma$ and $\Delta^{*}(s)$ is a small punctured disc about $s$.

This is of course exactly our situation - at the cocycle level a parabolic cocycle is one which satisfies the extra condition that

$$
\left[m_{\gamma}, n_{\gamma}\right]=\left[a_{1}, a_{2}\right]\left(M_{\gamma}-I\right)
$$

for some $\left[a_{1}, a_{2}\right] \in \mathbf{C}^{2}$ (possibly depending on $\gamma$ ) whenever $\gamma \in \Gamma\left(\cong \pi_{1}\right)$ is parabolic.

As a parabolic element corresponds to a multiple of a circuit around one of our missing points, it is easy to see that in terms of extensions, we have a locally split extension and conversely. Thus

$$
H_{\mathrm{para}}^{1} \cong H^{1}\left(X, R^{1} \pi_{*} \mathrm{C}\right)
$$

corresponds to locally split extensions which is isomorphic to $H_{\mathrm{DR}}^{1}$.

It only remains to determine the Hodge filtration on $H_{\mathrm{DR}}^{1}$ and we will be ready to do some examples.

3. The filtration. Now that we have identified $H^{1}\left(X, R^{1} \pi_{*} \mathbf{C}\right)$ with $H_{\mathrm{DR}}^{1}$ it is important to know what the Hodge filtration given by the Hodge structure on $H^{1}\left(X, R^{1} \pi_{*} \mathrm{C}\right)$ looks like in $H_{\mathrm{DR}}^{1}$. The Hodge decomposition of $H^{1}\left(X, R^{1} \pi_{*} \mathrm{C}\right)$ is constructed in Zucker [19] and it is shown to coincide with the one induced from $H^{2}(E, \mathbf{C})$ via the Leray spectral sequence. Moreover Hoyt [7] and Cox and Zucker [1] have given an interpretation of the Hodge filtration levels as spaces of Hoyt's generalized automorphic forms. Our goal is to identify these filtration levels in $H_{\mathrm{DR}}^{1}$. As we are interested in using this information for the calculation of 
Picard numbers, we will only sketch the results. Further details can be found in Stiller [17], Hoyt [7], and Cox and Zucker [1].

As before we fix our model (2.1) of $E^{\text {gen }}$ over $K(X)$. We denote the resulting Picard-Fuchs equation by $\Lambda$ and the space of locally exact elements in $K(X)$ by $L_{\Lambda}^{\text {para }}$. Our goal is to define two divisors $\mathfrak{A}_{0} \leq \mathfrak{A}$ on $X$, in terms of the local behavior of $\Lambda$, with the property that the (sub-) linear systems

$$
L\left(\mathfrak{H}_{0}\right) \cap L_{\Lambda}^{\text {para }} \subset L\left(\mathfrak{U}_{0}\right) \subset K(X)
$$

and

$$
L(\mathfrak{U}) \cap L_{\Lambda}^{\text {para }} \subset L(\mathfrak{U}) \subset K(X)
$$

consist of locally exact elements which are never exact and which correspond to the levels of Hodge filtration,

$$
H^{2,0} \text { and } H^{2,0} \oplus H^{1,1}
$$

respectively, on $H^{1}\left(X, R^{1} \pi_{*} \mathrm{C}\right) \cong H_{\mathrm{DR}}^{1}$. This will give us a set of unique representatives for the elements in these levels of the Hodge filtration.

In order to define $\mathfrak{A}_{0}$ and $\mathfrak{A}$, we introduce a local normal form for a locally exact equation

$$
\Lambda f=Z, \quad Z \in L_{\Lambda}^{\text {ara }} .
$$

At a point $p \in X$ where $\Lambda$ has exponents $r, s ; r \leq s$ (necessarily $r$, $s \in \mathbf{Q}$ ), choose a local parameter $t$. We first express (3.1) in terms of $t$

$$
\Lambda_{t} f=Z\left(\frac{d x}{d t}\right)^{2}
$$

and then "twist" by $t^{-[r]}$, where $[r]$ is the greatest integer $\leq r$, to get:

$$
\Lambda_{t^{-[r]}}\left(t^{-[r]} f\right)=t^{-[r]} Z\left(\frac{d x}{d t}\right)^{2} \quad \text { (see page } 163 \text { above) }
$$

where $\Lambda_{t^{-[r]}}$ now has the normalized exponents $r_{\text {norm }}=r-[r] \leq s_{\text {norm }}=s$ $-[r]$ with $0 \leq r_{\text {norm }}<1$. The expression $(*)$ is called the local normal form for $\Lambda f=Z$ at $p \in X$.

The possibilities for $r_{\text {norm }}$ and $s_{\text {norm }}$, together with the resulting fiber type (Kodaira [9]), are given in tables below. This list can be obtained by simple calculation using the explicit form of $\Lambda$ given in Stiller [15]. In addition, for $p \in X$ we define two numerical quantities, $a_{p}$ and $b_{p}$, for later use. They depend on the local normal form of $\Lambda$ at the chosen point $p \in X$. A quantity $i_{p}$ is also defined and its meaning explained, but no use will be made of this particular item. It is included merely for the sake of completeness. 

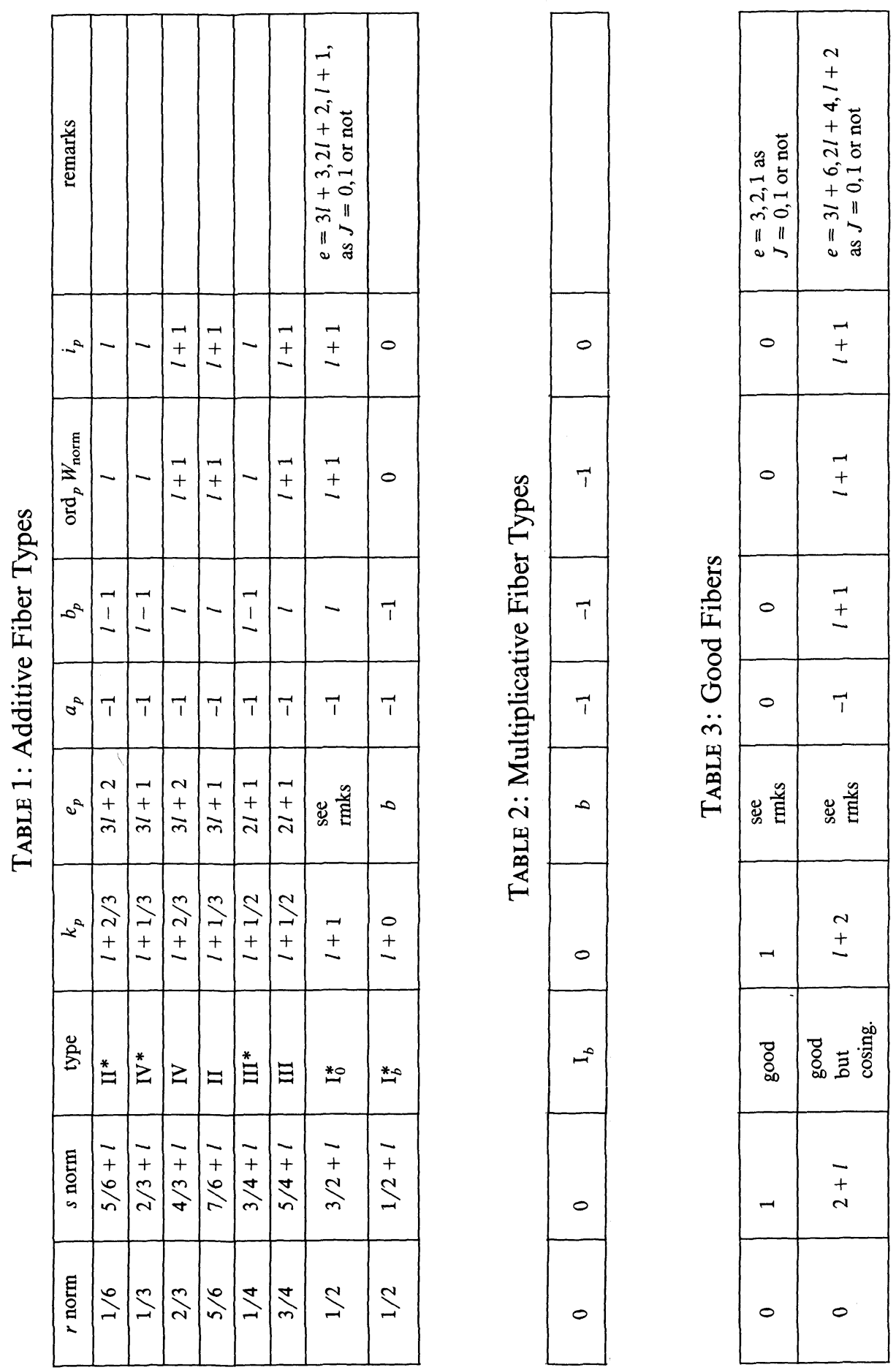
where

$$
l \in \mathbf{Z}, l \geq 0,
$$

$r_{\text {norm }} \leq s_{\text {norm }}$ are the normalized exponents at $p \in X$;

type is just the fiber type as designated by Kodaira in [9];

$k_{p}=s-r=s_{\text {norm }}-r_{\text {norm }}$ is the exponent difference at $p$;

$e_{p}$ is the ramification of $\mathscr{J}$, the functional invariant (Kodaira [9]), at $p$ viewed as a map $X \stackrel{\mathscr{J}}{\rightarrow} \mathbf{P}_{\mathbf{C}}^{1}$;

$\operatorname{ord}_{p} W_{\text {norm }}$ is the order of the Wronskian $W_{\text {norm }}$ of $\Lambda_{t^{-[r]}}$ which is easily seen to be $t^{-2[r]} W(d x / d t)$ where $W$ is the Wronskian of $\Lambda$;

$i_{p} \quad$ is called the index at $p$-it represents the dimension contributed to the 1, 1-part of $H^{1}\left(X, R^{1} \pi_{*} \mathrm{C}\right)$ i.e.

$$
\operatorname{dim}_{\mathbf{C}} H^{1,1}=\sum_{p \in X} i_{p} \text {. }
$$

DeFInItion 3.1. We define two divisors $\mathfrak{U}_{0}$ and $\mathfrak{A}$ by:

$$
\begin{aligned}
& \operatorname{ord}_{p} \mathfrak{U}_{0}=-[r]+\operatorname{ord}_{p}(d x)^{2}-b_{p} \\
& \operatorname{ord}_{p} \mathfrak{U}=-[r]+\operatorname{ord}_{p}(d x)^{2}-a_{p}
\end{aligned} \quad \text { for } p \in X,
$$

where $r$ is the smaller exponent of $\Lambda$ at $p$. For $Z \in L\left(\mathfrak{A}_{0}\right)=\{Z \in K(X)$ s.t. $\left.\operatorname{div} Z+\mathfrak{A}_{0} \geq 0\right\}$ we have ord ${ }_{p} t^{-[r]} Z(d x / d t)^{2} \geq b_{p}$ and similarly for $Z \in L(\mathfrak{A})$. This in turn allows us to define two linear systems

$$
\begin{aligned}
& L_{\Lambda}^{\text {para }}\left(\mathfrak{U}_{0}\right)=L\left(\mathfrak{A}_{0}\right) \cap L_{\Lambda}^{\text {para }}, \\
& L_{\Lambda}^{\text {para }}(\mathfrak{U})=L(\mathfrak{U}) \cap L_{\Lambda}^{\text {para }},
\end{aligned}
$$

by taking the locally exact elements in $L\left(\mathfrak{U}_{0}\right)$ and $L(\mathfrak{U})$ respectively.

We have the following facts:

Proposition 3.2. (i) $L\left(\mathfrak{U}_{0}\right) \subset L_{\Lambda}^{\text {para }}$ (ii) $L_{\Lambda}^{\text {para }}(\mathfrak{A}) \cap \Lambda K(X)=0$. In particular no element in $L_{\Lambda}^{\text {para }}(\mathfrak{U})$ or $L_{\Lambda}^{\text {para }}\left(\mathfrak{A}_{0}\right)=L\left(\mathfrak{A}_{0}\right)$ is exact.

Proof. The proof of (i) is quite easy. We consider

$$
\Lambda f=Z \text { for } Z \in L\left(\mathfrak{A}_{0}\right) \text {. }
$$

By Proposition 2.5 above, we must show that for every $p \in X$ and every single-valued solution $\tilde{\omega}$ of $\Lambda(\Lambda \tilde{\omega}=0)$ at $p$ the differential

$$
\frac{\tilde{\omega} Z}{W} d x
$$


has zero residue. Choose a local parameter $t$ at $p \in X$. We then have

$$
\frac{\tilde{\omega} Z}{W} d x=\frac{\left(t^{-[r]} \tilde{\omega}\right)\left(t^{-[r]} Z\left(\frac{d x}{d t}\right)^{2}\right)}{t^{-2[r]} W \frac{d x}{d t}} d t .
$$

Observe that $t^{-[r]} \tilde{\omega}$ is a single-valued solution to $\Lambda_{t^{-[r]}}\left(\right.$ i.e. $\Lambda_{t^{-[r]}} t^{-[r]} \tilde{\omega}=0$ ) so $\operatorname{ord}_{p} t^{-[r]} \tilde{\omega} \geq r_{\text {norm }} \geq 0$ at $p$. Also $t^{-2[r]} W(d x / d t)=W_{\text {norm }}$ the Wronskian of $\Lambda_{t^{-[r]}}$, so its order can be found in the tables above. Finally because $Z \in L\left(\mathfrak{A}_{0}\right)$ we have ord $t^{-[r]} Z(d x / d t)^{2} \geq b_{p}$.

As a single-valued solutions $\tilde{\boldsymbol{\omega}}$ exist only at points where the local monodromy is $\left(\begin{array}{ll}1 & 0 \\ 0 & 1\end{array}\right)$ or conjugate to $\left(\begin{array}{ll}1 & b \\ 0 & 1\end{array}\right), b>0$, a simple computation using the entries in Tables 2 and 3 shows that in these cases

$$
\frac{\tilde{\omega} Z}{W} d x
$$

is actually holomorphic at $p$. This proves (i).

To prove (ii), we assume for some $Z \in L_{\Lambda}^{\text {para }}(\mathfrak{A})$ that there exists $Z^{\prime} \in K(X)$ with $\Lambda Z^{\prime}=Z$.

Claim. For any point $p \in X$ ord $_{p} Z^{\prime} \geq r$ where $\Lambda$ has exponents $r \leq s$ at $p$.

In order to verify this, we work locally. Choose a local parameter $t$ at $p \in X$ and observe that $\Lambda Z^{\prime}=Z$ implies that

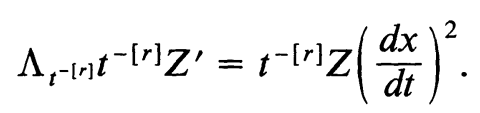

We consider two cases. First, take the case of a good fiber or a fiber of type $I_{b}, b \geq 1$, at $p$. From Tables 2 and 3 we see that

$$
\operatorname{ord}_{p} t^{-[r]} Z\left(\frac{d x}{d t}\right)^{2} \geq-1 \text {. }
$$

On the other hand $\Lambda_{t^{-[r]}}$ looks alike

$$
\frac{d^{2}}{d t^{2}}+\left(\frac{1-r_{\text {norm }}-s_{\text {norm }}}{t}+\cdots\right) \frac{d}{d t}+\left(\frac{r_{\text {norm }} s_{\text {norm }}}{t^{2}}+\cdots\right) .
$$

If $\operatorname{ord}_{p} t^{-[r]} Z^{\prime}$ were negative then the $d^{2} / d t^{2}$ term would force

$$
\Lambda_{t^{-[r]}} t^{-[r]} Z^{\prime}
$$

to have too large a pole. Thus ord $t^{-[r]} Z^{\prime} \geq 0$ from which it follows that ord $Z^{\prime} \geq[r]$. However, in the case we are considering, the exponents $r, s$ of $\Lambda$ are integers, and so $r=[r]$. In the case of an additive fiber type at 
$p \in X$, we again use the facts that

$$
\Lambda_{t^{-[r]}} t^{-[r]} Z^{\prime}=t^{-[r]} Z\left(\frac{d x}{d t}\right)^{2}
$$

and

$$
\Lambda_{t^{-[r]}}=\frac{d^{2}}{d t^{2}}+\left(\frac{1-r_{\text {norm }}-s_{\text {norm }}}{t}+\cdots\right) \frac{d}{d t}+\left(\frac{r_{\text {norm }} s_{\text {norm }}}{t^{2}}+\cdots\right) .
$$

As in the first case above, Table 1 shows that

$$
\operatorname{ord}_{p} t^{-[r]} Z\left(\frac{d x}{d t}\right)^{2} \geq-1
$$

from which it follows that $t^{-[r]} Z^{\prime}$ must be holomorphic at $p$. However, in the additive case both $r_{\text {norm }}$ and $s_{\text {norm }}$ are non-zero. Thus the final term in $\Lambda_{t^{-[r]}}$, namely $\left(r_{\text {norm }} s_{\text {norm }} / t^{2}+\cdots\right)$, actually has a pole of order 2 . If $t^{-[r]} Z^{\prime}$ did not vanish at $p$, then $\Lambda_{t^{-[r]}} t^{-[r]} Z^{\prime}$ would have a second order pole which contradicts (1). Thus

$$
\operatorname{ord}_{p} t^{-[r]} Z^{\prime} \geq 1 \text { and } \operatorname{ord}_{p} Z^{\prime} \geq 1+[r]>r .
$$

This establishes the claim.

To finish the proof we use the trivial fact that the sum of the lower exponents of $\Lambda$ is $>0$, namely

$$
\sum_{p \in X} r_{p}=\frac{\mu}{12} \quad \text { where } \mu=\text { degree of the map } X \stackrel{\mathscr{J}}{\rightarrow} \mathbf{P}_{\mathbf{C}}^{1} .
$$

We conclude that $\operatorname{div} Z^{\prime}$ would have to have positive degree- a contradiction.

We now state the main fact of this section:

Proposition 3.3. The subspaces of $H_{\mathrm{DR}}^{1}$ determined by the linear systems $L_{\Lambda}^{\text {para }}(\mathfrak{A})$ and $L\left(\mathfrak{A}_{0}\right)=L_{\Lambda}^{\text {para }}\left(\mathfrak{A}_{0}\right)$ correspond to the first $\left(H^{2,0} \oplus\right.$ $\left.H^{1,1}\right)$ and second $\left(H^{2,0}\right)$ levels of the Hodge filtration on $H^{1}\left(X, R^{1} \pi_{*} \mathrm{C}\right)$ via the natural isomorphism between $H^{1}\left(X, R^{1} \pi_{*} \mathrm{C}\right)$ and $H_{\mathrm{DR}}^{1}$.

Proof. The $(2,0)$-part of $H^{1}\left(X, R^{1} \pi_{*} \mathrm{C}\right)$ is readily identified with $H^{0}\left(E, \Omega_{E}^{2}\right)$, the space of global holomorphic two forms on $E$. We shall sketch here the correspondence between $L\left(\mathfrak{U}_{0}\right)$ and $H^{0}\left(E, \Omega_{E}^{2}\right)$.

Let $S \subset X$ denote the support of the singular fibers of $\pi: E \rightarrow X$. We set $X_{0}=X-S$ and $E_{0}=\pi^{-1}\left(X_{0}\right)$. Choose a base point $x_{0} \in X_{0}$ (which is not a cosingular point for $\Lambda$ ) and a basis of solutions $\omega_{1}, \omega_{2}$ to $\Lambda f=0$ 
which gives precisely the periods of $d X / Y$. In particular we will have Im $\omega_{1} / \omega_{2}>0$ on $X_{0}, J\left(\omega_{1} / \omega_{2}\right)$ will be the functional invariant $\mathscr{J}$ where $J$ is the elliptic modular function, and the monodromy representation $\pi_{1}\left(X_{0}, x_{0}\right) \rightarrow \mathrm{SL}_{2}(\mathrm{Z})$ will be the homological invariant of Kodaira [9]. Note that $\omega_{1}, \omega_{2}$ will perhaps be meromorphic on $X_{0}$, but that the quotient $\omega_{1} / \omega_{2}$ will be holomorphic.

We recall Kodaira's construction of $E$ over $X$ from [9]. First one constructs $E_{0}$ over $X_{0}$ and then compactifies.

The universal cover of $X_{0}$ will be the complex upper-half-plane $\mathfrak{h}$ and we define an action on $\mathfrak{h} \times \mathbf{C}$ by

$$
\begin{array}{lll}
\mathfrak{h} \times \mathbf{C} & \stackrel{\left(\gamma, n_{1}, n_{2}\right)}{\rightarrow} & \mathfrak{h} \times \mathbf{C} \\
(z, t) & \rightarrow & \left(\gamma z,\left(c_{\gamma} \omega(z)+d_{\gamma}\right)^{-1}\left(t+n_{1} \omega(z)+n_{2}\right)\right)
\end{array}
$$

where $\gamma \in \Gamma \subset \operatorname{PSL}_{2}(\mathbf{R})$ corresponds to an element of $\pi_{1}\left(X_{0}, x_{0}\right) ; n_{i} \in \mathbf{Z}$; $\omega(z)$ is the period function obtained by lifting $\omega(x)=\omega_{1}(x) / \omega_{2}(x)$ to the upper-half-plane via the universal covering map $\mathfrak{h}, z_{0} \rightarrow X_{0}, x_{0}$; and finally

$$
\left(\begin{array}{ll}
a_{\gamma} & b_{\gamma} \\
c_{\gamma} & d_{\gamma}
\end{array}\right)
$$

is the monodromy of $\omega_{1}, \omega_{2}$ around the path corresponding to $\gamma$.

This turns out to be a group action and the quotient is easily seen to give $E_{0}$ over $X_{0}$.

We consider now the multivalued meromorphic differential forms on $X_{0}$

$$
\frac{-\omega_{2} Z}{W} d x \quad \text { for } Z \in L\left(\mathfrak{U}_{0}\right)
$$

where $W=\omega_{1}\left(d \omega_{2} / d x\right)-\omega_{2}\left(d \omega_{2} / d x\right)$ is the Wronskian. A local calculation (see the proof of Proposition 3.2) shows that this differential is actually holomorphic on $X_{0}$. We pull it back to $\mathfrak{h}$ and wedge with $d t$

$$
\frac{-\omega_{2} Z}{W} d x \wedge d t
$$

to get a holomorphic two form on $\mathfrak{h} \times \mathbf{C}$ which is easily seen to be invariant under the action. It therefore gives a holomorphic two form on $E_{0}$. We now use Kodaira's description of the compactification, and in particular the local coordinates he gives, to check that the form extends to a holomorphic form on all of $E$. This requires the use of the estimates on 
the order of pole for $Z \in L\left(\mathfrak{A}_{0}\right)$. (The calculations for fiber types IV*, $I_{b}, I_{b}^{*}$ are carried out in Shioda [14].) The converse is also true because any holomorphic two form on $E$ when restricted to $E_{0}$ necessarily takes the form $h(z) d z \wedge d t$ because of invariance under elements of the form $\left(1, n_{1}, n_{2}\right)$. The function

$$
h(z) \frac{d z}{d x} \cdot \frac{W}{\omega_{2}}, \quad W, x, \omega_{2} \text { pulled-back to } \mathfrak{h},
$$

on $\mathfrak{h}$ is $\Gamma$-invariant and the resulting single-valued function on $X_{0}$ is easily checked to be in $L\left(\mathfrak{U}_{0}\right)$.

For $L_{\Lambda}^{\text {para }}(\mathfrak{A})$ the proof is more involved (see Stiller [17]) and we content ourselves with the remark that the normal function

$$
\Lambda \int_{\mathscr{O}}^{\mathscr{P}} \frac{d X}{Y}=Z \in K(X)
$$

for $\mathscr{P} \in E^{\text {gen }}(K(X))$ can be shown to have $Z \in L_{\Lambda}^{\text {para }}(\mathfrak{A})$ by local consideration of the singularities of $\Lambda$ (see Stiller [17]) and to correspond to the $(1,1)$-class given by $\left[(\mathscr{P})-(\mathcal{O})+\sum_{s \in S} D_{s}\right]$ where the correction factors are as mentioned in Proposition 1.5 above, and $\mathscr{P}$ is thought of as a section of $E$ over $X$.

One can also prove this result using Hoyt's generalized automorphic forms - see Shioda [14], Hoyt [6], Cox and Zucker [1] and Endo [3] for this interpretation.

Lastly we remark that if we have a morphism of another curve $X^{\prime}$ onto $X$

$$
X^{\prime} \stackrel{f}{\rightarrow} X
$$

then for the elliptic surface $E^{\prime}$ induced by pulling $E$ back via $f$, the resulting maps on cohomology (expressed as $H_{\mathrm{DR}}^{1}$ ) are easily determined. [The reader should note that $E^{\prime}$ is not $E \times{ }_{X} X^{\prime}$, but rather it is the relatively minimal compactification of the Néron model of the generic fiber of $E \times_{X} X^{\prime}$ over Spec $K\left(X^{\prime}\right)$. Because of this, there may not in general be a regular map from $E^{\prime}$ to $E$ over $f$, but there are maps induced on $H_{\mathrm{DR}}^{1}$ and $H^{2}$, as a simple Mayer-Vietoris argument shows.] We will examine this in the particular case where $X^{\prime}=X, f$ is an automorphism, and $E^{\prime} \cong E$ in the next section. 
4. Examples. In order to compute Picard numbers we will need to consider the effect of an automorphism of $E$ on $H_{\mathrm{DR}}^{1}$. Let $\phi$ be an automorphism of the base curve $X$ which is compatible with an automorphism $\Phi$ of $E$ which preserves the action

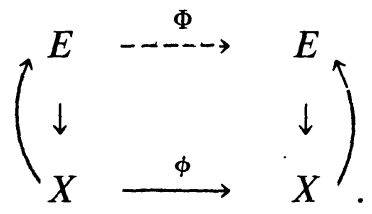

Specifically, if $E$ is given by a Weierstrass model

$$
Y^{2}=4 X^{3}-g_{2} X-g_{3}
$$

then $g_{2}, g_{3}$ should be invariant under the action $\phi^{*}: K(X) \rightarrow K(X)$ induced by $\phi$ on the function field of $X$. $\Phi$ will in general identify the (good) fiber over $x \in X$ with the (good) fiber over $\phi(x) \in X$ by either the identity or by the involution $Y \mapsto-Y$. These are the only possibilities because generically there is no complex multiplication.

In computing the Picard numbers in the examples below, it is immaterial whether $\Phi$ is taken to act as the identity or the involution in the fiber. We shall usually take it to be the identity. Also $\Phi$ need not be everywhere regular, but it should be regular after removing a finite number of fibers supported over a finite set $S \subset X$ with the property that $\phi(S)=S$. In all of our examples below however, $\Phi$ is everywhere regular. One that we shall shortly analyze is

$$
Y^{2}=4 X^{3}-\frac{27}{x^{12 k}} X-\frac{27}{x^{12 K}}
$$

with $\phi: x \rightarrow \zeta x$ over the $x$-sphere $\mathbf{P}_{\mathbf{C}}^{1}$ where $\zeta=e^{2 \pi i / 12 k}$.

Such automorphisms $\Phi$ occur in a natural setting. Given $E$ over $X$, suppose that $\left(\begin{array}{rr}-1 & 0 \\ 0 & -1\end{array}\right)$ is not in the global monodromy group $M \subset \mathrm{SL}_{2}(\mathbf{Z})$ and that $X$ is Galois over the $J$-line or Galois over the modular curve $X_{M}=\mathfrak{h}^{*} / M$ where $\mathfrak{h}^{*}$ is the extended upper half-plane $\mathfrak{h} \cup \mathbf{Q} \cup\{i \infty\}$. ( $X_{M}$ is the natural image for the period map on $X$ given by $\omega=\omega_{1} / \omega_{2}$, where $\omega_{i}$ are suitable solutions to $\Lambda$, the Picard-Fuchs equation; see Stiller [16].) We have

$$
X \stackrel{\omega}{\rightarrow} X_{M}
$$


and it is known that $E$ over $X$ is then induced by pulling-back the canonical elliptic modular surface over the modular curve $X_{M}$. Elements of the Galois group of $X$ over $X_{M}$ will then induce the desired type of automorphism.

Now because $\Phi: E \rightarrow E$ is compatible with an automorphism of the base it determines an automorphism

$$
\Phi^{*}: H_{\mathrm{DR}}^{1} \rightarrow H_{\mathrm{DR}}^{1}
$$

which clearly preserves Hodge type and is defined over $\mathbf{Q}$ :

$$
H^{1}\left(X, R^{1} \pi_{*} \mathbf{Q}\right) \subset H^{1}\left(X, R^{1} \pi_{*} \mathbf{C}\right) \cong H_{\mathrm{DR}}^{1} .
$$

More simply stated, $\Phi^{*}$ gives an automorphism of the rational cohomology $H^{2}(E, \mathbf{Q})$ which preserves the Hodge decomposition $H^{2,0} \oplus H^{1,1} \oplus$ $H^{0,2}$ of $H^{2}(E, \mathrm{C})$. We happen to be dealing with only a particular portion of the cohomology (see the remarks preceding Proposition 1.2 above) where a similar statement holds. In the examples, we shall examine the eigenspaces (which will turn out to be one-dimensional) and the $\mathbf{Q}$-irreducible subspaces for the action of $\Phi^{*}$ on $H_{\mathrm{DR}}^{1}$ and thereby determine the Picard number. The technique is best illustrated by specific examples.

Our first set of examples was originally discussed by Sasai (Sasai [12]). In his paper he computes the global monodromy representation. The base curve $X$ will be the sphere and we will denote the parameter by $x$ (i.e. $X$ is the $x$-sphere). A model for $E$ over $K(X)=\mathbf{C}(x)$ is given by

$$
Y^{2}=4 X^{3}-\frac{27}{x^{12 k}} X-\frac{27}{X^{12 k}} \quad k \geq 1 .
$$

Let $\zeta$ be the primitive $12 k$ th root of unity $e^{(2 \pi i / 12 k)}$. One sees immediately that (1) defines an elliptic surface over $X-\{0, \infty\}$ whose singular fibers are of type $I_{1}$ at $\xi^{i}, i=0, \ldots, 12 k-1$. The fibers over 0 and $\infty$ are good elliptic curves.

Proposition 4.1. For the model (1) above the operator annihilating the periods of $d X / Y$ is:

$$
\Lambda=\frac{d^{2}}{d x^{2}}+\frac{\left(1-x^{12 k}\right)-12 k}{x\left(1-x^{12 k}\right)} \frac{d}{d x}+\frac{\left(27 k^{2}+4 k^{2} x^{12 k}\right)}{x^{2}\left(1-x^{12 k}\right)} .
$$

Proof. Calculation (see Stiller [15] for the notation, terminology, and the formula for such equations). 
This equation is regular except at:

(i) 0 where $\Lambda$ has exponents $3 k, 9 k$;

(ii) $\infty$ where $\Lambda$ has exponents $-2 k, 2 k$;

(iii) $\zeta^{i}, i=0, \ldots, 12 k-1$, where $\Lambda$ has exponents 0,0 .

The elliptic surface corresponding to the model 1) has geometric genus $p_{g}=k-1$, irregularity $q=0$, and $\chi\left(\mathcal{O}_{E}\right)=k$. The monodromy representation is actually easy to compute (see Sasai [12]). The global monodromy group turns out to be $\mathrm{SL}_{2}(\mathbf{Z})$ and it follows that $E^{\text {gen }}(K(X))$, the group of $K(X)$-rational points on the generic fiber, is torsion free. We wish to prove:

THEOREM 4.2. Let $r$ denote the rank of the Mordell-Weil group $E^{\text {gen }}(\mathbf{C}(x))$ of $E^{\text {gen }}: Y^{2}=4 X^{3}-\left(27 / x^{12 k}\right) X-27 / x^{12 k}$ and let $\rho$ be the Picard number of the associated elliptic surface, then

$$
r=\sum_{\substack{d \mid 12 k \\ d \geq 1 \\ \text { admissible }}} \phi(d)=8+\sum_{\substack{d \mid 12 k \\ d \in\{7,8,10,15,18,20,42\}}} \phi(d),
$$

where $\phi$ is Euler's function, so $\phi(d)$ is the number of positive divisors of $d$ relatively prime to $d$, and $d$ is admissible if no primitive dth root of unity has argument between $\pi / 3$ and $\pi / 2$ inclusive. One can show that $d$ is admissible if and only if $d$ equals $1,2,3,7,8,10,12,15,18,20,42$. It follows that

$$
\rho=2+\sum_{\substack{d \mid 12 k \\ d \geq 1 \\ d \text { admissible }}} \phi(d)=10+\sum_{\substack{d \mid 12 k \\ d \in\{7,8,10,15,18,20,42\}}} \phi(d)
$$

Note $\rho \leq 58(r \leq 56)$ with equality if and only if $210 \mid k$.

We postpone the proof for a moment to examine $H_{\mathrm{DR}}^{1}$ in more detail. Further results of this sort for other surfaces appear in the next section. We begin by considering $H_{\mathrm{DR}}^{1}$ and the spaces $L_{\Lambda}^{\text {para }}(\mathfrak{U}), L\left(\mathfrak{U}_{0}\right)$.

Recall that given a section of $E$ over $X$ or equivalently a $K(X)$-rational point $(X(x), Y(x))$ on the generic fiber of $E / X$, we can produce an element of $K(X)$ via Manin's map (see Stiller [18])

$$
\begin{aligned}
& E^{\operatorname{gen}}(K(X)) \rightarrow K(X) \\
& \mathscr{P}=(X(x), Y(x)) \rightarrow \Lambda \int_{0}^{\mathscr{P}} \frac{d X}{Y}=\Lambda \int_{(0: 1: 0)}^{(X(x): Y(x): 1)} \frac{d X}{Y} .
\end{aligned}
$$

This map gives an injective homomorphism from the group of sections (there is no torsion in this instance) to the additive group $K(X)$ and the 
image lies in $L_{\Lambda}^{\text {para }}(\mathfrak{A})$ where

$$
\mathfrak{U}=\sum_{k i=0}^{12 k-1}(1) \zeta^{i}+(-3 k+1) 0+(2 k-3) \infty
$$

(see Tables 2 and 3 and Definition 3.1 above). A typical element of $L(\mathfrak{A})$ can be written

$$
Z=\frac{x^{3 k-1}(\text { polynomial of degree } \leq 11 k-2)}{\left(1-x^{12 k}\right)} .
$$

In order for $Z$ to be in $L_{\Lambda}^{\text {para }}(\mathfrak{U}) \subset L(\mathfrak{U})$ the residue conditions must hold at the cosingular points $0, \infty$. Near $x=0$ this is the condition that

$$
\frac{\omega Z}{W} d x, \quad W \text { the Wronskian, }
$$

have zero residue for any solution $\Lambda \omega=0$ at 0 . Now up to a constant

$$
W=c \frac{d \mathscr{J} / d x}{\mathscr{J}}=c \frac{12 k x^{12 k-1}}{\left(1-x^{12 k}\right)}, \quad c \neq 0,
$$

and so we must consider the differential

or

$$
\omega\left(\frac{\text { polynomial of degree } \leq 11 k-2}{(c 12 k) x^{9 k}}\right) d x
$$

$$
\omega \frac{p(x)}{c 12 k x^{9}} d x, \quad \operatorname{deg} p(x) \leq 11 k-2 \text {. }
$$

Near $x=0$ the differential equation can be seen to have a basis of solutions of the form

$$
\begin{aligned}
& x^{3 k}\left(1+c x^{12 k}+\cdots\right) \\
& x^{9 k} \text { (holomorphic). }
\end{aligned}
$$

As a result, if

$$
p(x)=a_{11 k-2} x^{11 k-2}+\cdots+a_{0}
$$

we must have $a_{6 k-1}=0$ to assure zero residues at $x=0$. A similar calculation at $\infty$ shows we must have $a_{7 k-1}=0$ to assure zero residues at $x=\infty$.

Thus a typical element of $L_{\Lambda}^{\text {para }}(\mathfrak{A}) \subset L(\mathfrak{A})$ is of the form $\frac{x^{3 k-1}\left(a_{11 k-2} x^{11 k-2}+\cdots+a_{7 k} x^{7 k}+a_{7 k-2} x^{7 k-2}+\cdots+a_{6 k} x^{6 k}+a_{6 k-2} x^{6 k-2}+\cdots+a_{0}\right)}{\left(1-x^{12 k}\right)}$. 
Next we determine the space of elements of the "first kind" which is $L\left(\mathfrak{U}_{0}\right)$ (see Definition 3.1 above). In this example we get

$$
\mathfrak{U}_{0}=\sum_{i=0}^{12 k-1}(1) \zeta^{i}+(-9 k+1) 0+(-2 k-3) \infty .
$$

And a typical element $Z \in L\left(\mathfrak{A}_{0}\right)$ is of the form

$$
\frac{x^{3 k-1}\left(a_{7 k-2} x^{7 k-2}+\cdots+a_{6 k} x^{6 k}\right)}{\left(1-x^{12 k}\right)},
$$

that is

$$
\frac{x^{9 k-1}(\text { polynomial of degree } \leq k-2)}{\left(1-x^{12 k}\right)} .
$$

We can now give the proof of Theorem 4.2.

Proof. Consider the automorphism $\Phi: E \rightarrow E$ compatible with the map $x \rightarrow \zeta x, \zeta=e^{2 \pi l / 12 k}$ on the base curve $X \cong \mathbf{P}_{\mathbf{C}}^{1}$. Explicitly, $E$ minus the fibers over 0 and $\infty$ is cut out in $(\mathbf{C}-\{0\}) \times \mathbf{P}_{\mathbf{C}}^{2}$ by the equation $0=-Z Y^{2}+4 X^{3}-\left(27 / x^{12 K}\right) X Z^{2}-\left(27 / x^{12 k}\right) Z^{3}$ and $\Phi$ is given by $(x ; X: Y: Z) \stackrel{\Phi}{\mapsto}(\zeta x ; X: Y: Z)$. This $\Phi$ actually extends to a regular automorphism of $E$ which when restricted to the fiber over 0 or $\infty$ is a complex multiplication. The resulting automorphism on cohomology, in particular on $H^{2}(E, \mathrm{C})$, preserves the Leray filtration (recall that the Leray spectral sequence for $\pi: E \rightarrow X$ and the constant sheaf $\mathbf{C}$ on $E$ degenerates at $E_{2}^{p q}$ ) and the Hodge decomposition.

We shall analyze this automorphism in terms of the action on $L_{\Lambda}^{\text {para }}(\mathfrak{A}) \subset H_{\mathrm{DR}}^{1}$. This action is easily seen to be

$$
\begin{array}{ll}
L_{\Lambda}^{\text {para }}(\mathfrak{H}) & \rightarrow L_{\Lambda}^{\text {para }}(\mathfrak{H}) \\
Z(x) & \mapsto Z(\zeta x) \zeta^{2} \quad \zeta=e^{2 \pi i / 12 k}
\end{array}
$$

(the extra factors of $\zeta$ are because one should work with $Z(d x)^{2}$ in order to get a parameter invariant description).

One could trace back through our description of the parabolic cohomology class determined by a locally exact equation to see that this corresponds to the action of our automorphism on the 2,0 and 1,1 parts of $E_{2}^{1,1}=H^{1}\left(X, R^{1} \pi_{*} \mathrm{C}\right)$, but the reader can see this in a more direct fashion. 
Suppose we consider an inhomogeneous equation

$$
\Lambda_{x} f(x)=Z(x), \quad Z \in L_{\Lambda}^{\text {para }}(\mathfrak{U}),
$$

where we have subscripted $\Lambda$ with an $x$ to indicate the parameter used in forming $\Lambda$

$$
\Lambda_{x}=\frac{d^{2}}{d x^{2}}+P(x) \frac{d}{d x}+Q(x) .
$$

Denote by $\Lambda_{\zeta x}$ the differential operator

$$
\Lambda_{\zeta x}=\frac{d^{2}}{d(\zeta x)^{2}}+P(\zeta x) \frac{d}{d(\zeta x)}+Q(\zeta x)
$$

and observe that in our case

$$
\Lambda_{\zeta x}=\zeta^{-2} \Lambda_{x}
$$

Viewing things on a suitable domain (e.g. slit the plane radially from the $12 k$ th roots of unity to $\infty)$, consider the functions $\omega_{i}(\zeta x)$ and $f(\zeta x)$. We have that $\omega_{1}\left(\zeta_{x}\right), \omega_{2}(\zeta x)$ are linear combinations of $\omega_{1}(x), \omega_{2}(x)$, because

$$
\Lambda_{x} \omega_{i}(\zeta x)=0
$$

and we see that

$$
\Lambda_{x} f(\zeta x)=\zeta^{2} \Lambda_{\zeta x} f(\zeta x)=\zeta^{2} Z(\zeta x)
$$

This describes the action of our automorphism. For example, consider the effect on $E^{\text {gen }}(\mathbf{C}(x))$-if $(X(x): Y(x): 1)$ is a solution to (1) above, then $(X(\zeta x): Y(\zeta x): 1)$ is also, and if

$$
\Lambda_{x} \int_{(0: 1: 0)}^{(X(x): Y(x): 1)} \frac{d X}{Y}=Z(x)
$$

we have

$$
\Lambda_{x} \int_{(0: 1: 0)}^{(X(\zeta x): Y(\zeta x): 1)} \frac{d X}{Y}=\zeta^{2} Z(\zeta x)
$$

because if we let

$$
f(x)=\int_{(0: 1: 0)}^{(X(x): Y(x): 1)} \frac{d X}{Y}
$$

be the normal function, then

$$
\int_{(0: 1: 0)}^{(X(\zeta x): Y(\zeta x): 1)} \frac{d X}{Y}=f(\zeta x) .
$$


Thus we have the diagram:

$$
\begin{array}{ccccccc}
(X(x): Y(x): 1) & \in & E^{\mathrm{gen}}(\mathbf{C}(x)) & \rightarrow & \mathbf{C}(x) & \ni & Z(x) \\
\downarrow & & \downarrow & & & & \downarrow \\
(X(\zeta x): Y(\zeta x): 1) & \in & E^{\mathrm{gen}}(\mathbf{C}(x)) & \rightarrow & \mathbf{C}(x) & \ni & Z(\zeta x) \zeta^{2}
\end{array}
$$

where the vertical left hand arrow is the map induced on sections by our automorphism and the horizontal arrows are Manin's map as described above.

Also consider the effect on two-forms. Recall that the space $L\left(\mathfrak{A}_{0}\right)$ can be identified with $H^{0}\left(E, \Omega^{2}\right)$ via

$$
Z \mapsto \frac{-\omega_{2} Z}{W} d x \wedge d t \quad \text { (see Proposition 3.3). }
$$

But remember that the Wronskian $W$ depends on the choice of derivation $d / d x$. A parameter free version would be

$$
\frac{-\omega_{2}(x) Z(x)(d x)^{2}}{W d x} \wedge d t
$$

Now $W(x) d x$ is invariant under $x \rightarrow \zeta x$ and we can see from the construction on pages 175-176 that $\omega_{2}(x)$ and $d t$ transform in essentially the opposite way: analytic continuation around $\gamma \in \pi_{1}\left(X_{0}, x_{0}\right)$ where $X_{0}=\mathbf{P}_{\mathbf{C}}^{1}-\{12 k$ th roots of unity $\}$ yields

$$
\begin{aligned}
\omega_{2}(x) & \mapsto\left(c_{\gamma} \omega(x)+d_{\gamma}\right) \omega_{2}(x) \\
d t & \mapsto\left(c_{\gamma} \omega(x)+d_{\gamma}\right)^{-1} d t+\text { other terms involving } d x
\end{aligned}
$$

Now $x \mapsto \zeta x$ is not, strictly speaking, monodromy, but it can be thought of in that way. It corresponds to a simple loop around 1 on the $J$-line, and we need only observe that the elliptic surface we are considering happens to be the pull-back (see pg. 176) of an elliptic surface over the $J$-line. In fact $\omega_{2}(x) d t$ is locally $\pm d X / Y$ in the fiber. It follows that the effect on 2-forms corresponds to the given action $Z(x) \mapsto Z(\zeta x) \zeta^{2}$ on $L\left(\mathfrak{A}_{0}\right) \subset$ $L^{\text {para }}(\mathfrak{A})$.

Now consider the eigenspaces for the action of our automorphism on $L_{\Lambda}^{\text {para }}$. All the eigenvalues are $12 k$ th roots of unity, and we see that the eigenspaces are all of the form

$$
\mathrm{C} \cdot \frac{x^{i}}{1-x^{12 k}}
$$


with eigenvalue $\zeta^{i+2}$. (This follows from our description of $L_{\Lambda}^{\text {para }}(\mathfrak{U})$ on page 180 and our description of the action of our automorphism on page 181.) We also get the important fact that the eigenvalues of our automorphism on $H^{1}\left(X, R^{1} \pi_{*} \mathrm{C}\right)$ occur with multiplicity one and all the eigenspaces are one dimensional. Now our automorphism preserves type, so the $(2,0),(1,1)$ and $(0,2)$ pieces are sums of distinct eigenspaces. The relevant picture is:

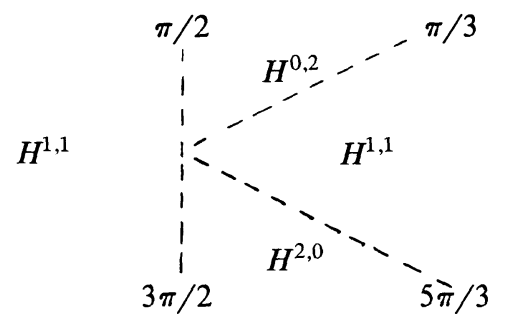

This diagram is arrived at by observing that the $(2,0)$ part, which corresponds to

$$
L\left(\mathfrak{A}_{0}\right)=\left\{\frac{x^{9 k-1}(\text { polynomial of degree } \leq k-2)}{1-x^{12}}\right\}
$$

is made up of the eigenspaces whose eigenvalues, $\zeta^{i}, i=9 k+1, \ldots, 10 k$ -1 , are precisely the $12 k$ th roots of unity whose arguments are between $3 \pi / 2$ and $5 \pi / 3$. Of course the $(0,2)$ part must lie in the conjugate region. This gives us the Hodge decomposition of $L_{\Lambda}^{\text {para }}(\mathfrak{U})$ into the $(2,0)$ part (namely $L\left(\mathfrak{A}_{0}\right)$ ) and the complementary $(1,1)$ part. As our automorphism preserves rational cohomology, it can be decomposed over $\mathbf{Q}$ into irreducible subspaces indexed by primitive $d$ th roots of unity for those $d \mid 12 k$. Now if the eigenspaces corresponding to all primitive $d$ th roots fall in $H^{1,1}$ then they span an irreducible rational subspace of dimension $\phi(d)$, where $\phi$ is Euler's function, and we have a rational component of $H^{1,1}$. The result follows.

\section{Additional examples.}

EXample 1. We consider over the sphere $\mathbf{P}_{\mathbf{C}}^{1}$ the elliptic surfaces $E_{k}$ given by

$$
E^{\text {gen }}: Y^{2}=4 X^{3}-3 x^{3 k} X-x^{5 k}, \quad k>0,
$$

which have functional invariant

$$
\mathscr{J}=\frac{1}{1-x^{k}} \quad \text { and } \quad \lambda^{2}=g_{2} / g_{3}=\frac{3}{x^{2 k}}
$$


The equation annihilating the periods of $d X / Y$ for this model of $E^{\text {gen }}$ is

$$
\Lambda=\frac{d^{2}}{d x^{2}}+\frac{(2 k+1) x^{k}-(k+1)}{x\left(x^{k}-1\right)} \frac{d}{d x}+\frac{35 k^{2} x^{k} / 36-27 k^{2} / 144}{x^{2}\left(x^{k}-1\right)} \text {. }
$$

Depending on the congruence of $k$ modulo 12 we have:

\begin{tabular}{|c|l|l|c|c|c|}
\hline$k=$ & $\begin{array}{l}\text { fiber } \\
\text { over } \infty\end{array}$ & $\begin{array}{l}\text { fiber } \\
\text { over } 0\end{array}$ & $\begin{array}{c}\text { fiber over } \\
k \text { th roots of } 1\end{array}$ & $p_{g}$ & $\rho$ \\
\hline $12 l+1$ & II & III* & $\mathrm{I}_{1}$ & $l$ & $r+9$ \\
$12 l+2$ & IV & $\mathrm{I}_{0}^{*}$ & $\mathrm{I}_{1}$ & $l$ & $r+8$ \\
$12 l+3$ & $\mathrm{I}_{0}^{*}$ & III & $\mathrm{I}_{1}$ & $l$ & $r+7$ \\
$12 l+4$ & $\mathrm{IV}^{*}$ & good & $\mathrm{I}_{1}$ & $l$ & $r+8$ \\
$12 l+5$ & $\mathrm{II}^{*}$ & III* & $\mathrm{I}_{1}$ & $l+1$ & $r+17$ \\
$12 l+6$ & good & $\mathrm{I}_{0}^{*}$ & $\mathrm{I}_{1}$ & $l$ & $\mathrm{r}+6$ \\
$12 l+7$ & II & III & $\mathrm{I}_{1}$ & $l$ & $r+3$ \\
$12 l+8$ & IV & good & $\mathrm{I}_{1}$ & $l$ & $r+4$ \\
$12 l+9$ & $\mathrm{I}_{0}^{*}$ & III* & $\mathrm{I}_{1}$ & $l+1$ & $r+13$ \\
$12 l+10$ & $\mathrm{IV}^{*}$ & $\mathrm{I}_{0}^{*}$ & $\mathrm{I}_{1}$ & $l+1$ & $r+12$ \\
$12 l+11$ & $\mathrm{II}^{*}$ & III & $\mathrm{I}_{1}$ & $l+1$ & $r+11$ \\
$12 l+12$ & good & good & $\mathrm{I}_{1}$ & $l$ & $r+2$ \\
\hline
\end{tabular}

$l \geq 0, l \in \mathbf{Z}$, where $r$ is the rank of the Mordell-Weil group $E^{\text {gen }}(\mathbf{C}(x))$ and $\rho$ is the Picard number.

In all cases it can be shown that

$$
r=\sum_{\substack{d \mid k \\ d \geq 1 \\ d \text { admissible }}} \phi(d)
$$

where $d$ admissible means that no primitive $d$ th root of unity has argument between $3 \pi / 2$ and $5 \pi / 3$ inclusive. For example, the surface is an elliptic $K 3$ surface for $k=5,9,10,11,13,14,15,16,18,19,20$, and

\begin{tabular}{|c|c|c|c|c|c|}
\hline$k$ & fiber type at 0 & fiber type at $\infty$ & fiber over $k$ th roots 1 & $r$ & $\rho$ \\
\hline 5 & III* & II* & $\mathrm{I}_{1}$ & 1 & 18 \\
\hline 9 & III* & $\mathrm{I}_{0}^{*}$ & $\mathrm{I}_{1}$ & 3 & 16 \\
\hline 10 & $\mathrm{I}_{0}^{*}$ & $\mathrm{IV}^{*}$ & $\mathrm{I}_{1}$ & 6 & 18 \\
\hline 11 & III & $\mathrm{II}^{*}$ & $\mathrm{I}_{1}$ & 1 & 12 \\
\hline 13 & III* & II & $\mathrm{I}_{1}$ & 1 & 10 \\
\hline 14 & $I_{0}^{*}$ & IV & $\mathrm{I}_{1}$ & 8 & 16 \\
\hline 15 & III & $\mathrm{I}_{0}^{*}$ & $\mathrm{I}_{1}$ & 11 & 18 \\
\hline 16 & good & IV* & $I_{1}$ & 6 & 14 \\
\hline 18 & $\mathrm{I}_{0}^{*}$ & good & $\mathrm{I}_{1}$ & 10 & 16 \\
\hline 19 & III & II & $\mathrm{I}_{1}$ & 1 & 4 \\
\hline 20 & good & IV & $\mathrm{I}_{1}$ & 14 & 18 \\
\hline 24 & good & good & $\mathrm{I}_{1}$ & 12 & 14 \\
\hline
\end{tabular}
24, and we have:

EXAMPLe 2. Over the $x$-sphere $\mathbf{P}_{\mathbf{C}}^{1}$ we consider the elliptic surfaces $E_{k}$, $k>0$, given in terms of a model for the generic fiber $E_{k}^{\text {gen }}$ over $\mathbf{C}(x)$ by:

$$
Y^{2}=4 X^{3}-3 x^{k} X-x^{2 k} \text {. }
$$


Depending on the congruence of $k$ modulo 12 we have:

\begin{tabular}{|c|l|l|c|c|c|}
\hline$k=$ & $\begin{array}{l}\text { fiber } \\
\text { over } \infty\end{array}$ & $\begin{array}{l}\text { fiber } \\
\text { over } 0\end{array}$ & $\begin{array}{c}\text { fiber over } \\
k \text { th roots of } 1\end{array}$ & $p_{g}$ & $\rho$ \\
\hline $12 l+1$ & IV* & III & $\mathrm{I}_{1}$ & $l$ & $r+9$ \\
$12 l+2$ & IV & $\mathrm{I}_{0}^{*}$ & $\mathrm{I}_{1}$ & $l$ & $r+8$ \\
$12 l+3$ & good & III* & $\mathrm{I}_{1}$ & $l$ & $r+9$ \\
$12 l+4$ & IV* & good & $\mathrm{I}_{1}$ & $l$ & $r+8$ \\
$12 l+5$ & IV & III & $\mathrm{I}_{1}$ & $l$ & $r+5$ \\
$12 l+6$ & good & $\mathrm{I}_{0}^{*}$ & $\mathrm{I}_{1}$ & $l$ & $r+6$ \\
$12 l+7$ & IV* & III* & $\mathrm{I}_{1}$ & $l+1$ & $r+15$ \\
$12 l+8$ & IV & good & $\mathrm{I}_{1}$ & $l$ & $r+4$ \\
$12 l+9$ & good & III & $\mathrm{I}_{1}$ & $l$ & $r+3$ \\
$12 l+10$ & IV* & $\mathrm{I}_{0}^{*}$ & $\mathrm{I}_{1}$ & $l+1$ & $r+12$ \\
$12 l+11$ & IV & III* & $\mathrm{I}_{1}$ & $l+1$ & $r+11$ \\
$12 l+12$ & good & good & $\mathrm{I}_{1}$ & $l$ & $r+2$ \\
\hline
\end{tabular}

$l \geq 0, l \in \mathbf{Z}$, and in all cases:

$$
r=\sum_{\substack{d \mid k \\ d \geq 1 \\ d \text { admissible }}} \phi(d)
$$

where $d$ admissible means that no primitive $d$ th root of unity has argument between $\pi / 2$ and $2 \pi / 3$ inclusive. For example, the resulting surface is an elliptic $K 3$ surface for $k=7,10,11,13,14,15,16,17,18$, $20,21,24$ and the resulting Picard numbers are $\rho=16,18,12,10,16,10$, $14,6,18,16,14$ respectively because $r=1,6,1,1,8,1,6,1,10,14,13,12$. (Note that for $k$ even these surfaces are isomorphic to the ones in Example 1 above. The reader may wonder when $k$ is even why the eigenvalues have changed sign:

Example 1

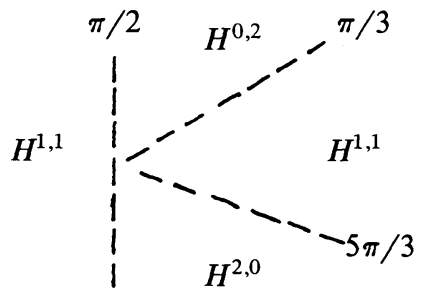

$3 \pi / 2$ versus

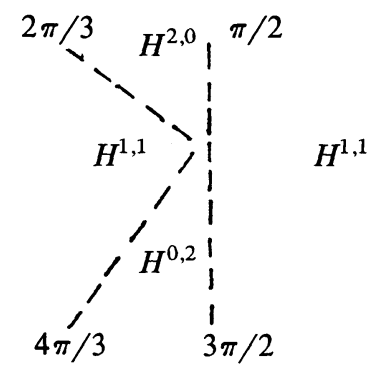

The reason is that after identifying the models for the generic fibers, one finds the maps $\Phi$ differ by the involution $X \rightarrow X, Y \rightarrow-Y$.)

EXAMPle 3. Over the $x$-sphere $\mathbf{P}_{\mathbf{C}}^{1}$ we consider the elliptic surfaces $E_{k}$ $k>0$ given in terms of a model for the generic fiber $E^{\text {gen }}$ over $\mathbf{C}(x)$ by:

$$
Y^{2}=4 X^{3}-3 x^{3 k}\left(x^{k}-8 / 9\right) X+x^{4 k}\left(x^{2 k}-\frac{4}{3} x^{k}+8 / 27\right) .
$$


Depending on the congruence of $k$ modulo 3 we have:

\begin{tabular}{|c|c|c|c|c|c|c|}
\hline$k=$ & $\begin{array}{c}\text { fiber } \\
\text { over } 0\end{array}$ & $\begin{array}{c}\text { fiber } \\
\text { over } \infty\end{array}$ & $\begin{array}{c}\text { fibers over } \\
k \text { th roots } \\
\text { of } 1\end{array}$ & $p_{g}$ & $\begin{array}{c}r \text { rank of } \\
\text { Mordell-Weil } \\
\text { group }\end{array}$ & $\begin{array}{c}\rho \text { Picard } \\
\text { number }\end{array}$ \\
\hline $3 l+1$ & $\mathrm{IV} *$ & $\mathrm{I}_{3 k}$ & $\mathrm{I}_{1}$ & $l$ & $\begin{array}{l}0 \text { if } l \text { even } \\
1 \text { if } l \text { odd } \\
1 \text { if } l \text { even } \\
0 \text { if } l \text { odd } \\
0 \text { if } l \text { even } \\
1 \text { if } l \text { odd }\end{array}$ & $\begin{array}{c}9 l+10 \text { if } l \text { even } \\
9 l+11 \text { if } l \text { odd } \\
9 l+9 \text { if } l \text { oden } \\
9 l+10 \text { if } l \text { even } \\
9 l+11 \text { if } l \text { odd }\end{array}$ \\
\hline
\end{tabular}

$l>0, l \in \mathbf{Z}$. Note that $\mathscr{J}=729 x^{k}\left(x^{k}-8 / 9\right)^{3} / 64\left(x^{k}-1\right)$. Also, $E_{1}$ is the elliptic modular surface for $\Gamma_{1}(3)=\left\{\left(\begin{array}{cc}a & b \\ c & d\end{array}\right) \in \mathrm{SL}_{2}(\mathbf{Z})\right.$ s.t. $a \equiv d \equiv$ $1 \bmod 3$ and $c \equiv 0 \bmod 3\}$ and $E_{3}$ is a rational elliptic modular surface for some $\Gamma^{\prime}(\neq \Gamma(3))$ of index 3 in $\Gamma_{1}(3)$.

EXAMPLE 4. We consider surfaces $E_{k}, k \in \mathbf{Z}, k>0$ given in terms of a model for the generic fiber over $\mathbf{C}(x)$ by

$$
Y^{2}=4 X^{3}-3 x^{4 k} X+x^{5 k}\left(x^{k}-2\right) .
$$

If we write $k=6 l+r$ with $0<r \leq 6$, then the geometric genus is $p_{g}=l$ and the surface has a singular fiber of type $I_{k}$ over $\infty$, singular fibers of type $I_{1}$ over the $k$ th roots of unity, and a singular fiber of type II (resp. IV *, I ${ }_{0}^{*}, \mathrm{IV}, \mathrm{II}$, good) over 0 as $k \equiv 1$ (resp. $2,3,4,5,0$ ) modulo 6 . One can show that for these surfaces we have the following:

\begin{tabular}{|c|c|c|c|c|c|}
\hline$k$ & $\begin{array}{c}\text { over } \\
\infty\end{array}$ & $\begin{array}{l}\text { over } \\
k \text { th } \\
\text { roots } \\
\text { of } 1\end{array}$ & $\begin{array}{r}\text { over } \\
0\end{array}$ & rank of Mordell-Weil group & $\begin{array}{l}\text { Picard } \\
\text { number }\end{array}$ \\
\hline $\begin{array}{l}6 l+1 \\
l \geq 0\end{array}$ & $\mathrm{I}_{k}$ & $\mathrm{I}_{1}$ & II* & $r=\left\{\begin{array}{l}4 \text { if } l \equiv 4 \bmod 5 \\
0 \text { otherwise }\end{array}\right.$ & $\rho=\left\{\begin{array}{l}k+1 \\
k+9\end{array}\right.$ \\
\hline $\begin{array}{l}6 l+2 \\
l \geq 0\end{array}$ & $\mathrm{I}_{k}$ & $\mathrm{I}_{1}$ & IV* & $r=\left\{\begin{array}{l}7 \text { if } 3 l+1 \equiv 0 \bmod 10 \\
5 \text { if } 3 l+1 \equiv 5 \bmod 10 \\
3 \text { if } 3 l+1 \equiv 2,4,6,8 \bmod 10 \\
1 \text { otherwise }\end{array}\right.$ & $\rho=\left\{\begin{array}{l}k=14 \\
k+12 \\
k+10 \\
k+8\end{array}\right.$ \\
\hline $\begin{array}{l}6 l+3 \\
l \geq 0\end{array}$ & $\mathrm{I}_{k}$ & $\mathrm{I}_{1}$ & $\mathrm{I}_{0}^{*}$ & $r=\left\{\begin{array}{l}6 \text { if } l \equiv 2 \bmod 5 \\
2 \text { otherwise }\end{array}\right.$ & $\rho=\left\{\begin{array}{l}k+11 \\
k+7\end{array}\right.$ \\
\hline $\begin{array}{l}6 l+4 \\
l \geq 0\end{array}$ & $\mathrm{I}_{k}$ & $\mathrm{I}_{1}$ & IV & $r=\left\{\begin{array}{l}7 \text { if } 3 l+3 \equiv 0 \bmod 10 \\
5 \text { if } 3 l+2 \equiv 5 \bmod 10 \\
3 \text { if } 3 l+2 \equiv 2,4,6,8 \bmod 10 \\
1 \text { otherwise }\end{array}\right.$ & $\rho=\left\{\begin{array}{l}k+10 \\
k+8 \\
k+6 \\
k+4\end{array}\right.$ \\
\hline $\begin{array}{l}6 l+5 \\
l \geq 0\end{array}$ & $\mathrm{I}_{k}$ & $\mathrm{I}_{1}$ & II & $r=\left\{\begin{array}{l}4 \text { if } l \equiv 0 \bmod 5 \\
0 \text { otherwise }\end{array}\right.$ & $\rho=\left\{\begin{array}{l}k+5 \\
k+1\end{array}\right.$ \\
\hline $\begin{array}{l}6 l+6 \\
l \geq 0\end{array}$ & $\mathrm{I}_{k}$ & $\mathrm{I}_{1}$ & $\begin{array}{l}\text { good, } \\
\text { but } \\
\text { cosing. }\end{array}$ & $r=\left\{\begin{array}{l}9 \text { if } l \equiv 9 \bmod 10 \\
7 \text { if } l \equiv 4 \bmod 10 \\
5 \text { if } l \equiv 1,3,5,7 \bmod 10 \\
3 \text { otherwise }\end{array}\right.$ & $\rho=\left\{\begin{array}{l}k+10 \\
k+8 \\
k+6 \\
k+4\end{array}\right.$ \\
\hline
\end{tabular}


Note: $p_{g}=l, q=0$ in all cases, and when $p_{g}=0$ we get $\rho=10$ as we should.

EXample 5. Again our base curve $X$ will be the sphere $\mathbf{P}_{\mathbf{C}}^{1}$ and we will denote the parameter by $x$ (i.e. $X \cong \mathbf{P}_{\mathbf{C}}^{1}$ is the $x$-sphere). We consider the elliptic surfaces $E_{k}, k \in \mathbf{Z}, k>0$ given in terms of a model for the generic fiber $E_{k}^{\text {gen }}$ over $\mathbf{C}(x)$ by

$$
Y^{2}=4 X^{3}-3 x^{12 k+3}\left(x^{4 k+1}-1 / 4\right) X-x^{20 k+5}\left(x^{4 k+1}+1 / 8\right) .
$$

These surfaces have singular fibers of type $I_{1}$ at the $4 k+1$ st roots of unity, $\mathrm{I}_{8 k+2}$ at $\infty$ and III* at 0 . The geometric genus is $p_{g}=k$ and one can show that the surface $E_{k}$ has Picard number

$$
\rho= \begin{cases}8 k+10, & k \equiv 0,1 \bmod 3, \\ 8 k+12, & k \equiv 2 \bmod 3,\end{cases}
$$

or equivalently the rank of the Mordell-Weil group $E_{k}^{\text {gen }}(\mathbf{C}(x))$ is

$$
r= \begin{cases}0, & k \equiv 0,1 \bmod 3 \\ 2, & k \equiv 2 \quad \bmod 3 .\end{cases}
$$

\section{REFERENCES}

[1] D. Cox and S. Zucker, Intersection numbers of sections of elliptic surfaces, Inv. Math., 53 (1979), 1-44.

[2] P. Deligne, Equations Différentielles a Points Singuliers Réguliers, Lec. Notes in Math., 163 Springer-Verlag, Berlin-Heidelberg-New York (1971).

[3] Y. Endo, Parabolic cohomologies and generalized cusp forms of weight three associated to Weierstrass equations over function fields, preprint.

[4] P. Griffiths, Differential Equations on Algebraic Varieties, Princeton lectures, unpublished.

[5] P. Griffith and J. Harris, Algebraic Geometry, John Wiley and Sons, New York, (1978).

[6] W. Hoyt, On Elliptic Surfaces and Automorphic Forms, preprint.

[7] _ An inhomogeneous Riemann-Roch theorem for generalized automorphic forms, preprint.

[8] N. Katz and T. Oda, On the differentiation of De Rham cohomology classes with respect to a parameter, J. Math. Kyoto Univ., (1968), 8-2.

[9] K. Kodaira, On compact analytic surfaces II, Ann. of Math., 77 (1963).

[10] , On compact analytic surfaces III, Ann. of Math., 78 (1963).

[11] A. Néron, Modéles minimaux des variétés abéliennes, Pub. Math. I.H.E.S., 21 (1964).

[12] T. Sasai, Monodromy representations of homology of certain elliptic surfaces, J. Math. Soc. Japan, 26, No. 2 (1974).

[13] G. Shimura, Introduction to the Arithmetic Theory of Automorphic Forms, Princeton University Press, Princeton (1971).

[14] T. Shioda, On elliptic modular surfaces, J. Math. Soc. Japan, 24, No. 1 (1972).

[15] P. Stiller, Differential equations associated with elliptic surfaces, J. Math. Soc. Japan, 32, No. 2 (1981). 
[16] Monodromy and invariants of elliptic surfaces, Pacific J. Math., 92, No. 2 (1981), 433-452.

[17] Automorphic forms attached to differential equations and relationships with the Picard number of an elliptic surface, I.H.E.S. preprint.

[18] Elliptic curves over function fields and the Picard number, Amer. J. Math. 102, No. 4 (1980), 565-593.

[19] S. Zucker, Hodge theory with degenerating coefficients: $L_{2}$ cohomology in the Poincare metric, Annals of Math., (1979).

Received August 28, 1985 and in revised form April 30, 1986. The author would like to thank the Institut des Hautes Études Scientifiques for its hospitality during the preparation of this paper.

Louisiana State University

BATON ROUGE, LA 70803-4918 



\section{PACIFIC JOURNAL OF MATHEMATICS EDITORS}

\author{
V. S. VARADARAJAN \\ (Managing Editor) \\ University of California \\ Los Angeles, CA 90024 \\ HERBERT Clemens \\ University of Utah \\ Salt Lake City, UT 84112 \\ R. FINN \\ Stanford University \\ Stanford, CA 94305
}

HERMANN FLASCHKA

University of Arizona

Tucson, AZ 85721

RAMESH A. GANGOLLI

University of Washington

Seattle, WA 98195

VAughan F. R. JONES

University of California

Berkeley, CA 94720

ROBION KIRBY

University of California

Berkeley, CA 94720
C. C. MoOrE

University of California

Berkeley, CA 94720

H. SAMELSON

Stanford University

Stanford, CA 94305

HAROLD STARK

University of California, San Diego

La Jolla, CA 92093

\section{ASSOCIATE EDITORS}
R. AREnS
E. F. BECKENBACH
B. H. NEUMANN
F. WOLF
K. YOSHIDA (1906-1982)

\section{SUPPORTING INSTITUTIONS}

UNIVERSITY OF ARIZONA

UNIVERSITY OF BRITISH COLUMBIA

CALIFORNIA INSTITUTE OF TECHNOLOGY

UNIVERSITY OF CALIFORNIA

MONTANA STATE UNIVERSITY

UNIVERSITY OF NEVADA, RENO

NEW MEXICO STATE UNIVERSITY

OREGON STATE UNIVERSITY
UNIVERSITY OF OREGON UNIVERSITY OF SOUTHERN CALIFORNIA STANFORD UNIVERSITY UNIVERSITY OF HAWAII UNIVERSITY OF TOKYO UNIVERSITY OF UTAH WASHINGTON STATE UNIVERSITY UNIVERSITY OF WASHINGTON 


\section{Pacific Journal of Mathematics}

\section{Vol. 128, No. $1 \quad$ March, 1987}

Anthony Peter Bahri and Peter Gilkey, The eta invariant, $\operatorname{Pin}^{c}$ bordism, and equivariant $\operatorname{Spin}^{c}$ bordism for cyclic 2-groups $\ldots \ldots \ldots \ldots \ldots \ldots 1$

Friedrich-Wilhelm Bauer, Extensions of generalized homology theories . . . 25

Marilyn Breen, A characterization theorem for compact unions of two

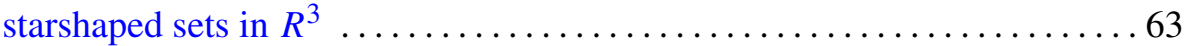

Boniface Ihemotuonye Eke, Special generating sets of purely inseparable extension fields of unbounded exponent $\ldots \ldots \ldots \ldots \ldots \ldots \ldots \ldots \ldots \ldots$

Robert William Gilmer, Jr. and William James Heinzer, Jónsson $\omega_{0}$-generated algebraic field extensions $\ldots \ldots \ldots \ldots \ldots \ldots \ldots \ldots \ldots . . .61$

Guido Lupacciolu, Holomorphic continuation in several complex variables

Douglas C. McMahon, Jaap C. S. P. van der Woude and Ta-Sun Wu,

Connectedness related to almost periodicity of compositions of flow

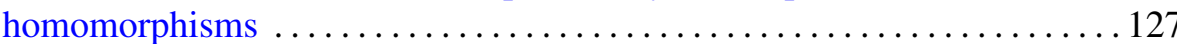

Sophocles Mercourakis, Some characterizations of analytic metric spaces

Peter Frederick Stiller, The Picard numbers of elliptic surfaces with many

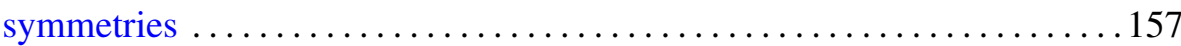

David J. Winter, Reducible complements of Lie algebra radicals ........ 191 Rade Živaljević, On a cohomology theory based on hyperfinite sums of microsimplexes 